\title{
Teoria dell' esplorazione del suolo ad impulsi di corrente a bassa frequenza
}

\author{
A. Belluigi \\ Ricevuto il 13 Giugno 1964
}

\section{PARTE}

Riassunto. - L'A. riesamina il problema dei "gap-potentials", o "potenziali lacunari " nei suoli uniformi, assoggettati a correnti pulsanti a bassa frequenza.

La memoria si divide in 2 parti. La la $^{\text {a }}$ consta di 5 capitoli: un primo introduttivo, un secondo critico, il terzo clie da una nuova valutazione dei potenziali scalare e indotto in un mezzo uniforme indefinito, valutazione che si sviluppa nei capitoli $4^{\circ}$ e $5^{\circ}$.

Nella $2^{\mathrm{a}}$ parte si tien conto dell'apporto della superficie del suolo, e i capitoli $6^{\circ}$ e $7^{\circ}$ offrono soluzioni del problema dei "potenziali lacunari" del tutto diverse da quelle finora apparse.

Summar. - The Author reexsamines the problem of the "gap-potentials " in the uniform ground, subjects to low-frequency current.

The paper is divided into two parts: the first is made up of 5 chapters, the Ist is introductive, the $2^{\text {nd }}$ critic, the $3^{\text {rd }}$ gives a new valutation of the scalar and induced potential in an indefinite uniform means; this valutation is developed in chapter $4^{\text {th }}$ and $5^{\text {th }}$.

In the second part the apport given by the surface of the ground is taken into account, and chapter $6^{\text {th }}$ and $7^{\text {th }}$ give some solutions of the "gap-potentials" problem, solutions which are absolutely different from those given up to now.

Résumé. - I'A. riexamine le probleme des "gap-potentials " dans les sols uniformes, avec des currents a tres basse frequence.

La memoire conste de 2 parties: la le $^{\text {o }}$ offre 5 chapitres, un premiere introductif, un $2^{e}$ critique, le troisieme donne une nouvelle valutation des potentials, scaler et induit, dans un milieux indefini uniforme, valutation qui se developpe dans les chapitres $4^{e}$ et $5^{e}$.

Dans la $2^{\mathrm{e}}$ partie on examine la contribution de la surface du sol, et consiste de 2 chapitres $\left(6^{\mathrm{e}}\right.$ e $7^{\mathrm{e}}$ ), et on parvient a des déductions nouvelles. 
§1. - a) Intendiamo riesaminare il problema dei "potenziali indotti, P.I., o potenziali provocati P.P.," in terreni omogenei isotropi a piccola conduttivita elettrica, eccitati con "impulsi di corrente a bassa frequenza ". A cio si riconnettono problemi vari, dalle ricerche dirette del petrolio (Elflex), alle ricerche dirette dell'acqua tellurica, o di altri "corpi geologici e minerali ».

Gli Autori che si sono occupati di questo indirizzo (Max, Müller, Evjen, Horton, Lewis, Belluigi, Vacquier, Buchheim, ece.) (1.6), pur impiegando sul tereno "correnti impulsive" discontinue (con misure sul terreno di gap-potential), hamo nel passato formulato teorie riguardanti lo studio del comportamento di correnti variabili periodiche continue a bassa frequenza, senza conseguire sviluppi teorici defmitivi.

A nostro avviso e l'Evjen $\left(^{7-8}\right)$ che meno s'e scostato da una aderente trattazione del problema, conseguenclo risultati qualitativi di una certa ronsistenza, anche se talora incerti.

A parte il ritorno d'interesse che il problema dei (P.P.) sta suscitando (v. Vachuier e sua Scuola), la questione offre tuttora aspetti teorici d'approfondimento.

Si sa quanta importanza abbia l'uso di "correnti variabili ", dato che quelle continue risentono solo della conduttivita $(\sigma)$ del terreno, e si sa come ci si debba limitare alle basse frequenze per evitare l'assorbimento conduttivo da parte del suolo (mezzo dissipativo).

Nei "sondaggi elettrici a bassa frequenza " interessa pero particolarmente l'uso di "impulsi discontinui di corrente" con pause, per misurare la reazione elettrica anche di strati molto profondi. Propagandosi tali impulsi con velocita finita (fatto fondamentale messo in evidenza dallo Horton), l'eliminazione d'arrivi di corrente, immediatamente dopo la trasmissione dell'impulso (raggi diretti, componenti varie parassite), dara via libera all'ottica del ritorno dell'moulso e.m. che può aver raggiunto notevoli profondita (Elflex). Come pure il filtraggio di altri percorsi d'onde e.m. potra permettere il rironoscimento di situazioni diverse.

Come corrente d'eccitazione l'Elflex impiega elettrodi soggetti ad impulsi altemati (+, e-), la mismra del potenziale alle sonde avviene negli intervalli in eni tale corrente viene interrotta, possono impiegarsi anche impulsi nello stesso verso, con forme diverse, periodici o meno.

La misura del "gap potential", (o del " potenziale lacunare", come noi preferiamo denominarlo) da, in sintesi, una somma di componenti (da separare e individualizzare con analisi teoriche), per un'adeguata e diretta interpretazione delle inomogeneita del suolo, che condizionano tali situazioni. 
L'Evjen ha dato una prima teoria approssimata specifica del "gap potential " relativa ad un suolo omogeneo, estesa con molte incertezze a qualche caso elementare d'inomogencita.

Comunque quest'indirizzo geoelettrico, con l'eliminazione di efletti parassiti di prossimita e di singolarita (dovuti anche alla corrente primaria) non potra non riuscire straordinariamente sensibile a qualsiasi anomalia insita nel terreno, ovunque ubicata, e per questo merita maggiore approfondimento teorico.

b) Ripercorriamo anzitutto, questa prima teoria, punto di partenza delle nostre ulteriori considerazioni sull'argomento.

Posto la "densita di corrente" $i=\sigma E$ ( $E=$ vettore elettrico), $\varepsilon$ costante dielettrica, lo "spostamento elettrico" $D=\varepsilon E, H=$ vettore magnetico ( $f / \partial t \sim f), \mu=$ permeabilità m., le equazioni di Maxwell, relative ad un mezzo indefinito omogeneo-isotropo si scrivono, con l'Evjen:

$$
\begin{aligned}
i=\operatorname{curl} H & =\dot{D}+4 \pi \alpha D, \\
\operatorname{curl} E & =-\mu \dot{H} . \\
\operatorname{div} \mu H & =0 \\
\operatorname{div} D & =4 \pi \Gamma,
\end{aligned}
$$

dove $\Gamma=$ densita spaziale della carica elettrica, $\alpha=\sigma / \varepsilon$, rapporto tra la conduttivita elettrica $\sigma$ e la $\varepsilon$, inverso del "tempo di relassazione" $\tau=\varepsilon / \sigma\left(\tau=2 \cdot 10^{-10}\right.$ sec. per l'acqua marina, $2 \cdot 10^{-6}$ sec. per l'acqua distillata).

Piu $\sigma$ e grande, piu $\tau$ e piccolo, e le cariche elettriche diventano rapidamente impercettibili, per cui è ovvio come riescano evanescenti i P.P., nei mezzi minerali metallici o conduttivi.

Come gia notato dal Planck, la costante $\varepsilon$ ha importanza prevalente per le oscillazioni sufficientemente lente, e per gli stati d'equilibrio.

Segue dalla 13], che esprime la continuita del flusso m., e l'assenzal dei poli $\mathrm{m}$. liberi (cariche libere), che il flusso $\mathrm{m}$. puo rappresentarsi notoriamente come il "curl" di un vettore arbitrario $A$, e poiche $\mu$ si considera costante, si ha:

$$
H=\operatorname{curl} A,(A=\text { "potenziale vettore m. ", p.v.m.) }
$$

Sostituendo la 1[5] nella 1[2], si ottiene l'c equazione fondamentale per l'esplorazione a bassa frequenza n:

$$
\operatorname{curl}(E+\mu \dot{A})=0
$$


il che implica

$$
E=-\nabla \phi+\mu \dot{A}
$$

poiche un vettore a rotazione nulla, deriva da un potenziale scalare.

Se le grandezze e.m. sono indipendenti dal tempo, curl $E$ e un rettore lamellare, e cioe $t=-\nabla \grave{\text { }}$. In prima approssimazione per frequenze estremamente basse, si puo ritenere: $A=0$.

La 1[7] indica che il vettore geo-elettrico $E$, in generale e il gradiente negativo d'un potenziale scalare ఏ, meno il prodotto della permeabilita m. $\mu$ per la derivata rispetto al tempo del p.v.m.: $\vec{A}$.

Sostituendo in $1[1]$ le 1[5] e 1[7] si ha notoriamente:

$\partial / \partial t \varepsilon \cdot[-\nabla \dot{\psi}-\mu \dot{A}]+4 \pi \alpha[-\nabla \dot{\phi}-\mu \dot{A}]=\operatorname{curl} \operatorname{curl} A=\operatorname{grad} \operatorname{div} A-\nabla^{2} A$.

Il p.v.m. e una soluzione dell'equazione delle onde:

$$
\mu \varepsilon \ddot{A}+4 \pi \mu \sigma A=\nabla^{2} \dot{A}
$$

se vale la condizione di Lorentz:

$$
4 \pi \sigma \phi+\varepsilon \dot{\phi}=-\operatorname{div} A \text {. }
$$

Applichiamo l'operatore div alla 1[7]:

$$
\operatorname{div} E-\operatorname{div} \nabla \phi=-\mu \mathrm{o} / \partial t \operatorname{div} A \text {. }
$$

La derivata rispetto al tempo della 1[9] da:

$$
4 \pi \sigma \mu \dot{\phi}+\varepsilon \mu \ddot{\phi}=-\jmath / \nu t \operatorname{div} A,
$$

da cui "l'equazione non omogenea delle onde ":

$$
\operatorname{div} E=-\nabla^{2} \dot{\phi}+4 \pi \sigma \mu \dot{\phi}+\mu \ddot{\phi} .
$$

La 1[10] dunque si ottiene dalla 1[7], tenendo presente la derivata rispetto al tempo della 1[9] (ch'e la condizione di Lorentz), affinche il p.v.m. sia una soluzione dell'equazione delle onde, purche la divergenza del vettore elettrico s'annulli:

$$
\operatorname{div} E=0 .
$$

Basato direttamente sull'equazione di campo di Maxwell, lo "spettro di frequenza estremamente basso " può essere ridotto, in prima approssimazione, alla semplice teoria del potenziale scalare.

La div della 1[1], valevole in un mezzo omogeneo risulta:

$$
\partial / \partial t \operatorname{div} D+t \pi \operatorname{div} \alpha D=0 ; \quad \dot{I}+4 \pi \alpha \Gamma=-\nabla \alpha \cdot D
$$


in quanto:

$$
\operatorname{div}(f \bar{v})=f \operatorname{div} \bar{v}+\operatorname{grad} f \cdot \bar{v}=f \operatorname{div} \bar{v}+\nabla f \cdot \bar{v} .
$$

Non si esclude inoltre che:

$$
\begin{aligned}
& \operatorname{div} D=\operatorname{div}(\varepsilon E)=\varepsilon \operatorname{div} E+E \operatorname{grad} \varepsilon ; \quad \operatorname{div} \varepsilon E=4 \pi T \\
& \partial / \partial t(\varepsilon \operatorname{div} E+E \operatorname{grad} \varepsilon)+4 \pi \alpha \cdot 1 / 4 \pi \operatorname{div} \varepsilon E=-\nabla \alpha \cdot(\varepsilon E) \\
& \partial / \partial t(\varepsilon \operatorname{div} E+E \nabla \varepsilon)+\alpha \varepsilon \operatorname{div} E+\alpha E \nabla \varepsilon=-\nabla \alpha \cdot(\varepsilon E)
\end{aligned}
$$

per cui quando $\nabla a=0$, o quando i parametri del mezzo sono costanti, la densitá di volume della carica elettrica, e quindi la div $E$ s'annullera esponenzialmente col tempo.

Se viceversa $a=\sigma / \varepsilon$ non $\dot{e}$ costante, il gradiente non s'anmulla, $e$ la 1[11] mostra che appariranno nel mezzo cariche spaziali.

In particolare se il mezzo ha una superficie di discontinuita in cui i parametri cambiano bruscamente da una parte e dallaltra (un insieme di valori da un lato, altro insieme dall'altro), la 111] mostra che appare una divergenza superficiale, cioe la divergenza puo essere nulla dappertutto, ma diversa da zero lungo la superficie.

Una densita superficiale di carica s'attiva lungo questo limite, e poiche qualsiasi irregolarita nel mezzo, in accordo con la 1[11], importa l'accumulo di cariche di volume quando una corrente l'attraversa, detta irregolartia provochera una "perturbazione potenziale" P.P.

$\S 2$ - Dopo un certo numero di considerazioni basate sull'equazioni di Maxwell, si mostra che ogni "irregolarita di terreno " provoca una formazione di cariche elettriche, a causa della variabilita di $\sigma$ e $\varepsilon$.

In un "mezzo omogeneo" una soluzione delle sucitate equazioni di Maxwell, è data secondo Tivjen da:

$$
\begin{aligned}
& \phi=\frac{J}{4 \pi \sigma} \cdot \frac{1}{1+j u} \cdot \frac{e^{-\omega_{1}}}{r} \cdot e^{-j\left(m_{2}\right.}{ }^{-2 \pi f t)}, \quad(j \text { è qui unita immaginaria }) 2[1] \\
& \omega_{1}=\omega^{2}\left(1 \overline{1+u^{2}}-u\right), \quad \omega_{2}^{2}=\omega^{2}\left(1 \overline{1+u^{2}}+u\right), \\
& \omega^{2}=2 \pi \sigma \mu \omega r^{2}=\text { quadrato della "distanza numerica } \text {. } \\
& u=\text { costante adimensionale }=\varepsilon f / 2 \sigma, \omega=2 \pi f \text {. }
\end{aligned}
$$

Ia parte reale della $\phi$ rappresenta il "potenziale scalare " dovuto alla corrente $J \cos 2 \pi f t$.

Per tener conto dell'influenza della superficie del terreno sarebbe necessario ricomere alla teoria ai Sommerfeld, ma date le complicazioni 
che essa comporta, ci si accontenta qui ai una approssimazione per basse frequenze. In seguito verà da noi ripreso in esame questo delicato punto, ripartendo proprio dalle posizioni di Sommerfeld, che secondo noi non si possono evitare.

Se per $f=0$ si ottiene $\dot{\phi}$ in superficie moltiplicando la $2[1]$ per un fattore 2 (il che fisicamente significa che il suolo si comporta come un riflettore perfetto), la stessa cosa e ammissibile (con le riserve dette) anche per le basse frequenze. In altre parole sulla superficie si può porre uguale a 2 volte la parte reale della $\phi$ cioe:

$$
\begin{aligned}
& \phi=\frac{J}{2 \pi \sigma}\left(F, \cos 2 \pi f t+F_{2} \operatorname{sen} 2 \pi f t\right) \\
& F_{1}=\frac{1}{1+u^{2}} \cdot \frac{e^{-\omega_{1}}}{r}\left(\cos \omega_{2}-u \operatorname{sen} \omega_{2}\right), \\
& F_{2}=\frac{1}{1+u^{2}} \frac{e^{-\omega_{2}}}{r} \quad\left(\operatorname{sen} \omega_{2}+u \cos \omega_{2}\right) .
\end{aligned}
$$

Nella prassi col "potenziale lacunare", la corrente d'alimentazione, non è però della forma $J \cos 2 \pi f t$.

Per ottenere $\phi$ bisognerebbe anzitutto decomporre questa corrente nelle sue componenti di Fourier, e ad ognuna di esse applicare la formula 2[2]. Si ritiene pero che gia il primo termine dia un'approssimazione sufficiente. Naturamente nella misura del "potenziale lacunare" viene eliminata la parte in fase con la corrente d'alimentazione, il che comporta che in questa approssimazione bisogna conservare solo il temine in sen $2 \pi f t$, quello cioe che si trova in quadratura con la corrente immessa nel suolo. Si perviene per il rapporto $Q$ dei valori medi del potenziale scalare e della intensità della corrente alimentante, all'espressione media approssimata:

$$
Q=F_{2} / 2 \pi \sigma
$$

Non viene precisato di che media si tratta nel rapporto $Q$, ma dato il carattere approssimato dei calcoli, non ha importanza se si usano medie aritmotiche o medic quadratiche.

La teoria e applicata a misure esegruite col classico metodo Wenner. Indicando con a la distanza delle $J$ onde, quindi $3 a$ quella degli elettrodi esterni, si ottiene allora dalla 2[3]:

$$
Q_{1}=\left[F_{2}(a)-F_{2}(2 a)\right] / \pi \sigma .
$$


Finora si e trascurato completamente il potenziale veltore $\vec{A}$, mir il suo contributo si puo valutare dalla teoria del dipolo, nel seguente modo:

$$
Q_{m}=-\frac{M I}{\pi \sigma} \omega^{2} \frac{e^{-\omega_{1}}}{r} \cos \omega_{2}
$$

ron $M$ (induzione mutua) costante di ordine di grandezza unitaria. La $Q$ totale, nel dispositivo, diverra allora:

$$
Q=Q_{1}+Q_{m}
$$

Data che tutta la teolia e stata impostata per basse frequenze, basta sviluppare la $Q$ in serie di potenze della "distanza numerican, cioe di $f^{1 / 2}$. Si perviene cosi ar mospressione del tipo:

$$
Q=\frac{f \cdot \sigma}{\pi \mu}\left(A_{1}+A_{2} f^{1 / 2}+A^{3} f+\cdots\right),
$$

dove i coefficienti di questo sviluppo $A_{1}, A_{2} \ldots$ dipendono dal distanziamento elettrodico a. Siceome e difficile che nella teoria approssimata Ie $A_{2}, A_{3} \ldots$, calcolate dalle formule precedenti, abbiano ma esatta dipendenza da $a$, si considera solo $A_{1}$. Questa dipendenza si trova facilmente dalle $2\left[2^{\prime}\right], 2[4], 2\left[4^{\prime}\right], 2[5]$. Basta porre:

$$
\begin{gathered}
\left.\operatorname{sen} \omega_{2}=\omega_{2}=\omega_{1}-\omega\right) \cdot e^{-\omega_{1}}=1-\omega_{1}=1-\omega, \\
\cdot \cos \omega_{2}=1,
\end{gathered}
$$

e si ottiene il primo termine della $2\left[5^{\prime}\right]$ :

$$
A_{1}=\varepsilon / 2 \mu \sigma^{2} a^{2}+1-\lambda I .
$$

Ripetendo l'esperienza con diversi valori di $f$, si ricavano quelli di $Q$ per un prefissato a. Da questi dati mediante la $2\left[5^{\prime}\right]$, si ricava col metodi dei minimi quadrati $A_{1}, A_{2}, A_{3} \ldots$

Lo stesso procedimento lo si ripete con altri valori di $a$. Le $A_{1}$, corrispontenti alle diverse $a$, vengono riportate su un grafico dove come ascisse sono scelte le grandezze $a^{-2}$. Se la teoria e approssimativamente corretta, questi dati dovrebbero giacere su una retta il cui coefficiente direttivo e dato da $\varepsilon / 2 \mu \sigma^{2}$. Noto $\sigma$ si puo ottenere la costante diclettricar relativa $\varepsilon$. Nell'esempio addotto effettivamente i punti sumnominati giaceiono su ma retta. Da questa retta si puo anche ricavare il valore di $M$ dalla 2[6] che risulta dell'ordine di grandezza dell'unita, il che confermerebbe la teoria. 
Questa conferma ha per noi valore dubbio, dato che $M$ non entra nell'espressione della $Q_{1} 2[4]$, e quindi non ha nulla a vedere con la teoria che conduce alla $2[3]$ e poi alla $2[4]$.

Inoltre si deduce un valore estremamente grande di $\varepsilon \sim 10^{8}$ che non si concilia evidentemente con lo sviluppo in serie $2\left[\breve{b}^{\prime}\right]$.

Questo eccessivo valore di $\varepsilon$ e ritenuto possibile, in quanto anche altri fattori sperimentali possono rendere plausibile una grandezza così alta, ma e difficile pronunciarsi in proposito, con argomenti simili. Solo si puo osservare che essendo il "potenziale lacunare" molto sensibile alla composizione del suolo in una teoria che tiene conto dell'inomogeneità, potrebbero comparire in $A_{1}$ termini del tipo $c / a^{2}$ che influiscono talmente da superare d'importanza il termine $\varepsilon / 2 \mu \sigma^{2} a^{2}$.

$\S 3$. - Indipendentemente dalle considerazioni precedenti del § 2 diamo ora una prima nostra valutazione approssimata del "potenziale scalare" in un mezzo omogeneo di piccola conduttivita, generato da un elettrodo al suolo che fornisce corrente impulsiva. Fermi restando i simboli precedenti, introduciamo la "velocita di segnale " o di propagazione $v=c / 1 / \mu \varepsilon$ nel mezzo considerato ( $c=$ velocita della luce).

S'e visto che la densita di carica $\Gamma$ soddisfa l'equazione:

$$
\dot{\Gamma}+4 \pi \alpha \Gamma=0,
$$

da cui

$$
\Gamma=\gamma e^{-4 \pi \alpha \Gamma}
$$

con $\gamma$ densita di carica per $t=0, \alpha=\sigma / \varepsilon$.

D'altronde il "potenziale scalare" $\phi$ soddisfa l'equazione:

$$
\Delta \phi=-\frac{\varepsilon \mu}{c^{2}} \underset{\mathrm{i}}{\ddot{\mathrm{r}}}-\frac{4 \pi \sigma \mu}{c^{2}} \underset{\mathrm{i}}{\dot{\mathrm{d}}}=-\frac{4 \pi}{\varepsilon} \Gamma,
$$

che in virtù della 1.[8] e con le posizioni di cui sopra, si puo scrivere nella forma:

$$
\Delta \phi-\frac{1}{v^{2}} \ddot{\phi}-\frac{4 \pi^{2}}{v^{2}} \alpha \dot{\phi}=-\frac{4 \pi}{\varepsilon} \gamma e^{-4 \pi u t} \text { per } t>0: \text {. }
$$

Supporremo ora a piccolo sufficientemente da poter trascurare grandezze dell'ordine di $\alpha^{2}$.

Posto:

$$
\oint=\psi \cdot e^{-2 \pi a t}
$$


la $3\left[3^{\prime}\right]$ diventa:

$$
\Delta \psi-\frac{1}{v^{2}} \ddot{\psi}+\frac{4 \pi^{2} \alpha^{2}}{v^{2}} \psi=-\frac{4 \pi}{\varepsilon} \gamma e^{-2 \pi a t} .
$$

Trascurando il termine in $\alpha^{2}$ la $3[5]$ diventa:

$$
\Delta \psi=\frac{1}{v^{2}} \ddot{\psi}-\frac{4 \pi}{\varepsilon} \gamma e^{-2 \pi \alpha t}
$$

che è della forma "dell'equazione d'onde ":

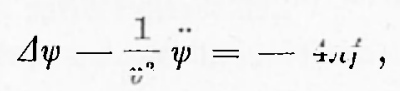

di cui e ben nota la soluzione mediante i "potenziali ritardati ".

Notiamo subito che non e sempre lecito trascurare grandezze dell'ordine di $a^{2}$, tanto che in una $2^{2}$ nostra valutazione, (meno approssimata s'intende), terremo conto di $\alpha^{2}$.

Nel caso di un mezzo omogeneo indefinito la predetta soluzione, per la formula di Kirchoff, e data dalla somma d'un intregrale di volume, e uno di superficie:

$$
\psi=\iint_{-\infty}^{+\infty} \varrho^{-1}[f]_{l-v / \varrho} d \xi d \eta d \zeta+\ldots
$$

La distanza $\varrho=\left[(x-\xi)^{2}+(y-\eta)^{2}+(z-\zeta)^{2}\right]^{1 / 2}$.

L'integrale superficiale, addendo omesso nella $3[7]$, ha valore identicamente nullo all'infinito, come si riconosce considerando la superficie limitante il mezzo, cosi lontana dall'emittore (con emissione solitaria ad es. rettangolare) che al tempo $t$ nessun punto e stato ancora raggiunto.

Il simbolo [ ] simnifica valori ritardati, e nella $f$ al posto di $t$ deve porsi $t-\varrho / v$.

Si abbia ora un elettrodo puntiforme nell'origine, che fornisca istantaneamente nell'istante $t=0$ la carica $q$.

Questo significa porre per la densita elettrica di carica

$$
\gamma=q \delta(\xi) \delta(\eta) \delta(\zeta)
$$

(sorgente in un elettrodo puntiforme, $\gamma=\infty$ all'origine), $\delta(\xi)$ indica la "funzione di Dirac", che come è noto, e ovunque zero e infinita per $\xi=0$, in maniera che:

$$
\int_{-\infty}^{+\infty} f(\xi) \delta(\xi) d \xi=f(0)
$$


e in generale:

$$
\llbracket \int_{1} G(x, y, z ; \xi, \eta, \zeta) \delta(\xi) \delta(\eta) \delta(\zeta) d x d y d z=G(x, y, z ; 0,0,0)
$$

Inoltre essendo per $t<0$ il campo uguale a zero, per la $f$ data dalla $3[6]$ bisogna porre:

$$
f<0,(\text { per } t<0) ; f=\frac{\ddot{q}}{\varepsilon} \delta(\xi) \delta(\eta) \delta(\zeta) e^{-2 \pi r a t} .
$$

Con questo valore di [f], la 3[7] ci fornisce immediatamente, ricordando la definizione della funzione di Dirac, i valori di:

$\left\{\begin{array}{l}\psi=0, \quad \text { (per } t<r / v), \quad \text { dove } r=\left[x^{2}+y^{\alpha}+z^{-1 / 2}\right. \\ \left.\psi=\frac{a}{\varepsilon r} \exp \cdot[-2 \pi \alpha(t-r / v)], \quad \text { (per } t<r / v\right) .\end{array}\right.$

Dalle $3[10]$ e $3[5]$ si ricava per la 0 :

$$
\left\{\begin{array}{l}
\phi=0, \quad(\text { per } t<r / v) \\
\phi=\frac{q}{\varepsilon r} \exp [-2 \pi \alpha(2 t-r / v)], \quad(\text { per } t>r / v) .
\end{array}\right.
$$

Se invece che nell'istante $t=0$, l'impulso arriva nell'istante $\tau$, allora basta nelle $3[11]$ sostituire $(t-\tau)$ al posto di $t$, e si perviene alle:

$$
\left\{\begin{array}{l}
\phi=0, \quad(\text { per } t-\tau<r / v) \\
\phi=\frac{q}{\varepsilon r} \exp [-2 \pi \alpha(2 t-2 \tau-r / v)], \quad(\text { per } t-\tau>r / v) .
\end{array}\right.
$$

Si abbia ora una durata dell'impulso $T$, cioe nell'intervallo $0<\tau<T$ venga fornita la carica $Q$ dalla corrente $J=Q / T$ (impulso rettangolare).

Per ottenere le formule relative a questa situazione, basta evidentemente sommare (intregrare) gli effetti dei singoli impulsi. Si perviene cosi all'espressione per il potenziale $p$ :

$$
\varphi=\int_{0}^{T} \phi(\tau) d \tau
$$

essendo $\phi$ dato dalla $3\left[11^{\prime}\right]$, ove al posto di $q$ si e posto $J=Q / T$.

Data la doppia definizione della $\phi$ (v. $3\left[11^{\prime}\right]$ ), occorre distinguere tre situazioni:

a) $t<r / v$, prima dell'arrivo dell'impulso alla distanza $r$,

b) $r / v<t<r / v+T$, momenti seguenti l'arrivo dell'inizio dell'impulso ma precedenti la sua fine,

c) $t>r / v+T$, momenti che seguono l'arrivo di tutto l'impulso. 
Nel caso a), essendo la $\phi(\tau)=0$ in tutto l'intervallo $0<\tau<T$, si ottiene $\varphi=0$.

In b) si ha $\phi(\tau)=0$ per $\tau>t-v / v$, e $\phi(\tau) \neq 0$ per $0<\tau<t-$ - $v / v+T$, quindi la $\varphi$ si calcola - v. 3[11'] e 3[12] - dalla:

$$
\varphi=\frac{J}{\varepsilon r} \cdot \int_{0}^{t-r / v} \exp [-2 \pi \alpha(2 t-2 \tau-r / v)] d \tau .
$$

Nel caso c) la $\phi(\tau)$ e $\neq 0$ in tutto l'intervallo fra 0 e $T$, quindi l'integrazione viene estesa a tutto l'intervallo.

La $\varphi$ di conseguenza si calcola dalla:

$$
\varphi=\frac{J}{\varepsilon r} \int_{0}^{T} \exp [-2 \pi \alpha(2 t-2 \tau-r / v)] d \tau .
$$

Eseguendo le integrazioni indicate, si perviene alle eseguenti espressioni per la $\varphi$ :

$$
\begin{gathered}
\varphi=0, \quad(\text { per } t<r / v) \\
\varphi=\frac{J}{4 \pi \sigma r}\left[e^{-2 \pi \alpha r / v}-e^{-2 \pi \alpha(t-r / v)}==\frac{J}{2 \pi \sigma r} \operatorname{sh} 2 \pi \alpha\left(t-\frac{r}{v}\right) \cdot e^{-2 \pi a t}\right. \\
(\operatorname{per} r / v<t<r / v+T)
\end{gathered}
$$

$\varphi($ gap-potential $)=\frac{J}{2 \pi \sigma r} \operatorname{sh} 2 \pi \alpha T e^{-2 \pi a(2 t-T-r / v)},($ per $t>r / v+T)$.

Si noti che per un valore fisso di $r$, la $\phi$ assume il valore zero fin all'istante $r / v$ (arrivo dell'inizio dell'impulso), cresce fino al momento $r / v+T$ (arrivo della fine dell'impulso), fino ad un massimo dato da:

$$
m_{m}=\frac{J}{2 \pi \sigma} \operatorname{sh} 2 \pi a T e^{-2 \pi a T} \cdot \frac{e^{-2 \pi a r / v}}{r},
$$

dopodiche continua a decrescere rapidamente fino allo zero.

§ 4. - Studiamo ancora, pii da vicino, il "potenziale scalare", generato da un "impulso rettangolare" in un mezzo infinito omogeneo, isotropo, uniforme.

Introduciamo la grandezza $\beta=2 \pi \sigma / \varepsilon$, inverso del doppio del "tempo di relassazione ". Com'e noto la densita di carica soddisfa la equazione differenziale:

$$
\frac{d \Gamma}{d t}+2 \beta \Gamma=0
$$


che risolta da per $\Gamma$ in funzione di $t$

$$
\Gamma=\Gamma_{\tau} e^{-2 \beta(t-\tau)},
$$

dove $\Gamma_{\tau}$ e la densita nel momento $\tau$.

Si considera anche qui una carica generata da una corrente $J$ nell'origine del sistema $0(x, y, z)$, che genera quindi, nei momenti $\tau,(\tau+d \tau)$, una densita di carica data da:

$$
\delta(x) \delta(y) \delta(z) J d \tau \quad \quad(\delta \text { funzione di Dirac). }
$$

Nel tempo $t$ ognuna di queste cariche, in virti della 4[2], dara un contributo:

$$
\delta(x) \delta(y) \delta(z) e^{-2 \beta(t-\tau)} \cdot d \tau,
$$

da cui s'ottiene per la densita $\Gamma$ :

$$
\Gamma=\delta x \delta y \delta z \cdot \quad \begin{array}{ll}
\left.\right|_{0} ^{t} e^{-2 \beta(t-\tau)} d \tau, & (\text { per } 0<t<T) \\
\int_{0}^{T} e^{-2 \beta(t-\tau)} d \tau, & (\text { per } t>T) .
\end{array}
$$

Eseguendo le integrazioni indicate si perviene per la $T$ :

$$
\Gamma=J \delta(x) \delta(y) \delta(z) \cdot f(t)
$$

con:

$$
f(t)=\left\{\begin{array}{lll}
\left(1-e^{-2 \beta t}\right) / 2 \beta & , & (\text { per } 0<t<T) \\
\frac{1}{\rho_{f}}\left(e^{-2 \beta T}-11 e^{-\beta t}\right. & , & (\text { per } t>T) .
\end{array}\right.
$$

Determinata $\Gamma$, passiamo a considerare il " potenziale scalare " $\varphi$ che soddisfa l'equazione:

$$
\Delta_{\digamma}-\frac{1}{v^{2}} \frac{\partial^{2} \varphi}{\partial t^{2}}-\frac{2 \beta}{v^{2}} \frac{\partial \varphi}{\partial t}=-\frac{4 \pi}{\varepsilon} \Gamma .
$$

Posto:

$$
\varphi=\frac{J}{\varepsilon} \psi \cdot e^{\beta t},
$$

a sostituendo a $l^{\prime}$ l'eguaglianza 4[4] si perviene per la $\psi$ alla:

$$
\Delta \psi-\frac{1}{\varkappa^{2}} \ddot{\psi}+\frac{\beta^{2}}{\eta^{2}} \psi=-4 \pi \delta(x) \delta(\eta) \delta(z) \cdot g(t),
$$


con:

$$
g(t)= \begin{cases}\frac{1}{2 \beta}\left(e^{\beta t}-e^{-\beta t}\right), & (\text { per } 0<t<T) \\ \frac{1}{2 \beta}\left(e^{2 \beta t}-1\right) e^{-\beta t}, & (\operatorname{per} t>T) .\end{cases}
$$

Si chiede ora una soluzione della 4[7] che si annulli per $r=\infty$, e che per $t=0$ si annulli insieme con la sua prima derivata parziale rispetto al tempo. Per risolvere questo problema ci serviremo del metodo della trasformazione di Laplace. Sia $\bar{\psi}$ la trasformata di Laplace della $\psi$, poniamo cioè:

$$
\bar{\psi}=\int_{0}^{\infty} \psi e^{-s t} d t
$$

La trasformata della $\ddot{\psi}$ e allora data (tenendo conto delle condizioni iniziali), da:

$\int_{0}^{\infty} \ddot{\psi} e^{-s t} d t=\left(\dot{\psi} e^{-s t}\right)_{r}^{\infty}-s \int_{0}^{\infty} \dot{\psi} e^{-s t} d t=s\left(\left.\psi e^{-s t}\right|_{v}+s \int_{0}^{\infty} \psi e^{-s t} \mathrm{dt}=s^{2} \bar{\psi} \cdot 4\left[8^{\prime}\right]\right.$

Inoltre la trasformata della $g(t)$ e data da:

$$
\begin{aligned}
\int_{0}^{\infty} g(t) e^{-s t} d t & =\frac{1}{2 \beta} \int_{e_{0}}^{T}\left(e^{\beta t}-e^{\beta t}\right) e^{-s t} d t+\left(e^{2 \beta t}-1\right) \int_{T}^{\infty} e^{-(\beta+s) t} d t= \\
& =\frac{1}{s^{2}-\cdot \beta^{2}}\left[1-e^{-(\delta-\beta) T}\right] .
\end{aligned}
$$

Moltiplicando ora la 4[7] per $\left(e^{-s t}\right)$ e integrando tra 0 e $\infty$, si perviene alla seguente equazione differenziale per la $\bar{\psi}$ :

$$
\Delta \psi-\frac{1}{v^{2}}\left(s^{2}-\beta^{2}\right) \bar{\psi}=-\frac{\Delta \pi}{s^{2}-\beta^{n}}\left[1-e^{-(s-\beta) T}\right] \delta(x) \delta(y) \delta(z), 4[9]
$$

di cui si richiede quella soluzione che si annulla all'infinito.

Ora per $s>\beta$, questa soluzione e nota, ed e data da:

$\bar{y}=\frac{1}{s^{2}-\beta^{2}}\left[1-e^{(s-\beta) T}\right] \frac{1}{\gamma} \cdot e^{-\sqrt{s^{2}-\beta^{2}}} \cdot r / v$, dove $r^{2}=x^{2}+y^{2}+z^{2} \cdot 4[10]$

Dalla $4[10]$ si puo, (mediante il teorema dell'inversione della trasformata di Iaplace), ottenere la $\psi$ :

$$
\psi=\frac{2 \pi \dot{j}_{j}}{1} \int_{\dot{L}} \bar{\psi}(s) e^{s t} d s
$$


dove $L$ e una retta parallela all'asse immagrinario, a destra del punto $s=\beta$. Sostituendo la $4[10]$ nella $4[11]$, e quest'ultima nella $4[6]$, si perviene per la $\varphi$ a:

$$
\varphi(r, t)=-\frac{J}{\varepsilon r}\{\mu(r, t)-\mu(r, t-T),
$$

con:

$$
\mu(r, t)=\frac{1}{2 \pi i} e^{-\beta t} \sum_{i} \frac{d s}{\left(s^{2}-\hat{p}^{2}\right)} \cdot e^{s t-\sqrt{s^{2}-\beta^{2}}(r / v)},
$$

dove con $1 \longdiv { s ^ { 2 } - \beta ^ { 2 } }$ e intesa quella determinazione del radicale che sul semiasse $s>\beta$ assume valori positivi.

Anzitutto si puo osservare che l'esponenziale nell'integrale 4[13] ha all'infinito, come termine principale, $s(t-r / v$ ) (essendo all'infinito $\left.1 / s^{2}-\beta^{2} \rightarrow s\right)$, quindi per $t<r / v$, si può deformare il cammino spostandolo verso destra, dove non esistono punti singolari. Questo significa che la $u(r, t)$ sara nulla per $t<r / v$ che corrisponde fisicamente al fatto che il segnale arriva con la velocita $v$. La 4[12] si puó scrivere dunque:

$$
\begin{array}{lll}
\varphi(r, t)=0 & , & \text { (per } t<r / v) \\
\varphi(r, t)=\frac{J}{\varepsilon r} u(r, t) & \text { (per } r / v<t<T+r / v) \\
\varphi(r, t)=\frac{I}{c t}[u(r, t-u(r, t-T)], & \text { per }(t>T+r / v) .
\end{array}
$$

In seguito nello studio della $u(r, t)$ possiamo perciò limitarci al caso $t>r / v$.

Anzitutto effettueremo nella $4[13]$ un cambiamento di variabile per renderla piu semplice. Posto:

$$
\begin{aligned}
& s=\frac{\beta}{2}(z / k+k / z), \quad 1 \overline{s^{2}-\beta^{2}}=\frac{\beta}{2}(z / k-k / z), \\
& k^{2}=(t-r / v) /(t+r / v), \quad a=\beta \sqrt{t^{2}-r^{2} / v^{2}},
\end{aligned}
$$

la $4[13]$ diventa:

$$
u(r, t)=\frac{k e^{-\beta t}}{\pi j \beta}\left\lceil\frac{d z}{i} \frac{a}{\left(z^{2}-k^{2}\right)}\left(z+\frac{1}{2}\right)\right.
$$

essendo ora $L$ una retta parallela all'asse immaginario a destra del punto $z=k$.

Essendo $a>0$, si puo sostituire questo cammino (deformandolo verso sinistra) con uno limitato e chiuso, che racchiude nel suo interno 
i punti $z=0 \pm 7$; questo percorso (preso in senso antiorario) s'indichera con $L^{\prime}$. Ora dalla teoria delle funzioni di Bessel e nota la formula:

$$
e^{\frac{a}{2}(a+z l / z)}=\underline{\Sigma}_{-\infty}^{+\infty} z^{n} I_{n}(a)
$$

dove $I_{n}(z)$ e la funzione di Bessel modificatal (o la funzione $j^{-n} J_{n}(j z)$ d'ordine $n$ ).

Per calcolare la $u(r, t)$ basta quindi sostituire la 4[17] nella 4[16], e integrare termine a termine lungo la curva chiusa $L^{\prime}$. Ora s'osservi che per $n \leqslant 0$ si ha:

$$
\frac{k}{\pi j} \int_{L^{\prime}}^{j} \frac{z^{n}}{z^{2}-k^{2}} d z=0
$$

ottenuto immediatamente sostituendo $L^{\prime}$ con un cerchio il cui raggio tende all'infinito. Per $n>0$ quest'integrale invece e uguale alla somma dei residui nei poli $k$ e $-k$, cioe si ha:

$$
\frac{k}{\pi j} \oint_{L^{\prime}} \frac{z^{n}}{z^{2}-i^{2}} d z=\frac{1}{2 \pi j} \oint_{L^{\prime}} \frac{z^{n}}{z-k} d z-\frac{1}{2 \pi j} \oint_{L^{\prime}} \frac{z^{n}}{z+k} d z=k^{n}-(-k)^{n} .
$$

Tenendo presente la $4[18]$ e la $4\left[18^{\prime}\right]$, si ottiene immediatamente, dalle $4[16]$ e $4[17]$ la:

$$
u(r, t)=2 \frac{e^{-\beta t}}{\beta} \sum_{n=0}^{\infty}\left(\frac{t-r / v}{t+r / v}\right)^{n+\frac{1}{2}} \cdot I_{2 n+1}\left(\beta \overline{t^{2}-\frac{v^{2}}{r^{2}}}\right) .
$$

La 4[19] è una serie che per valori non molto alti di $\beta \sqrt{t^{2}-r^{2} / v^{2}}$, converge benissimo. I valori di $I_{2^{n+1}}(a)$ si possono ricavare da quelli tabellati di $I_{o}(a)$ e $I_{\perp}(a)$, mediante le formule ricorrenti:

$$
\left\{\begin{array}{l}
I_{3}(a)=\left(1+8 / a^{2}\right) I_{1}(a)-\frac{4}{a} I_{0}(a) \\
I(2 n+1 a)=(2 n-1)\left[\frac{1}{n-1}+\frac{8 n}{a^{2}}\right] I_{2 n-1}(a)-\frac{n}{n-1} I_{2 n-3}(a) .
\end{array}\right.
$$

Le $4[20]$ si ottengono dalla nota relazione:

$$
I_{v-1}(a)-I_{\nu+1}(a)=\frac{2 v}{a} I_{v}(a) .
$$


La $1^{\mathrm{a}}$ delle $4[20]$ si ottiene applicandola per $v=1$ e per $v=2$, ed eliminando la $I_{2}$. La $2^{a}$ delle $4[20]$ si ottiene invece applicando la formula menzionata successivamente per $v=2 n-2,2 n-1,2 n$, ed eliminando le $I_{2 n}, I_{2 n-2}$. Del resto nelle tavole si trovino anche i valori di $I_{3}, I_{5}, I_{7}$ per cui la prima delle 4[20] non serve, mentre la seconda basta applicarla a partire da quel valore di $n$ per cui non esistono piu tabelle.

Per alti valori di $n$ basta per la $I_{n}$ usare l'asintotica:

$$
I_{n}(a)=\frac{a^{n}}{\sqrt{2 \pi}} \frac{e^{1 \overline{a^{2}+n^{2}}}}{\sqrt[4]{a^{2}+n^{2}}\left(a+\sqrt{\left.a^{-}+n^{2}\right)^{n}}\right.} .
$$

Per valori di a piuttosto alti, la 4[19] converge lentamente, ed è conveniente determinare un'espressione asintotica.

Si parte di nuovo dall'espressione $4[16]$, cambiando la variabile $z$ in:

Essa diventa allora:

$$
z=\zeta+\sqrt{\zeta^{2}-1} \text {. }
$$

$$
u(r, t)=\frac{k e^{-t \beta}}{\pi j \beta} \int_{\dot{L}} \frac{e^{a \zeta}}{\left[\left(1+k^{2}\right) \sqrt{\zeta^{2}-1}+\left(1-i^{2}\right) \zeta\right]^{2}} \cdot \frac{d s}{\sqrt{\zeta^{2}-1}},
$$

dove $L$ e una retta parallela all'asse imaginario a destra del punto $\zeta=1$, e $\sqrt{\zeta^{2}}-1$ indica quella radice che e positiva per valori reali di $\zeta>1$. In questo integrale si può deformare il cammino d'integrazione in uno chiuso, che aggrira in senso antiorario il segmento dcll'asse reale $-1<\zeta<+1$. In altre parole quest'integrale si puo scrivere come diflerenza di due integrali estesi all'intervallo $-1,+1$, il primo immediatamente al disotto dell'asse reale, dove $\sqrt{\zeta^{2}-1}=-\mathrm{j} \sqrt{1-\zeta^{2}}$, il secondo al di sopra dell'asse reale dove $\sqrt{\zeta^{2}-1}=j \sqrt{\zeta^{2}-1}$.

Si ottiene cosi per l'espressione integranda della 4[23]:

$$
\begin{aligned}
j^{-} & {\left[\frac{e^{a p}}{j\left(1+k^{2}\right) \sqrt{1-\zeta^{2}}+\zeta\left(1-h^{2}\right)} \cdot \frac{1}{-j \sqrt{1-\zeta^{2}}}-\right.} \\
& \left.-\frac{e^{a p}}{j\left(1+k^{2}\right) \sqrt{1-\zeta^{2}}+\left(1-h^{2}\right) \zeta} \cdot \frac{1}{j \sqrt{1-\zeta^{2}}}\right]= \\
& =\frac{2 k\left(1-k^{2}\right) \zeta}{\left(1+k^{2}\right)^{2}-4 k^{2} \zeta^{2}} \cdot \frac{e^{a \zeta}}{\sqrt{1-\zeta^{2}}}= \\
& =\frac{1-k^{2}}{2}\left[\frac{1}{1+k^{2}-2 k \zeta}-\frac{1}{1+h^{2}-2 h \zeta}\right] \cdot \frac{e^{a \zeta}}{\sqrt{1-\zeta^{2}}},
\end{aligned}
$$


e quindi per la $u(r, t)$ l'espressione:

$$
u(r, t)=\frac{(1-k-)^{-t \beta}}{2 \pi \beta} \int_{-1}^{+1}\left(\frac{1}{1+k^{2}-2 k \zeta}-\frac{1}{1+k^{2}+2 k \zeta}\right) \frac{e^{a \zeta}}{\sqrt{1-\zeta^{2}}} d \zeta .
$$

Posto ora:

la 4[25] diventa:

$$
\zeta=1-2 x^{2}, \quad \frac{1-k}{2 \sqrt{k}}=\lambda, \quad \frac{2 \sqrt{k}}{1+k}=x,
$$

$u(r, t)=\frac{e^{a-\beta t}}{\pi \beta}\left\{\frac{\lambda}{x} !_{-0}^{1} \frac{e^{-2 a x}}{\lambda^{2}+x^{2}} \cdot \frac{d x}{\sqrt{1-x^{2}}}-\operatorname{in} \int_{0}^{1} \frac{e^{-2 a x}}{\sqrt{1-\lambda^{2} x^{2}}} \cdot \frac{d x}{\sqrt{1-x^{2}}}\right\}$.

Osserviamo che per grandi valori di $a$ il contributo principale nei due integrali proviene dal punto $x=0$, dato che in questo caso il fattore esponenziale decresce molto rapidamente. Inoltre non avendo il limite superiore molta influenza, si trascurano le grandezze dell'ordine $e^{-2 a}$, e lo si puo quindi cambiare in $\int_{0}^{1} e^{-{ }^{\prime} a x} f(x) d x$ e in $\int_{0}^{\infty} e^{--a x} f(x) d x$.

Consideriamo anzitutto il secondo termine. Dopo quello che si e detto, si può sostituire il fattore:

$$
\begin{aligned}
& \frac{1}{1-\chi^{-x^{2}}} \cdot \frac{1}{\sqrt{1-x^{2}}} \text {, col suo sviluppo in serie di Taylor, ottenendo } \\
& \frac{1}{\left(1-x^{2}\right)^{1 / 2}}=\sum_{0}^{\infty} \frac{(2 n-1) ! !}{2^{n} n !} x^{2 n} ; \frac{1}{1-\chi^{-x^{2}}}=\sum_{0}^{\infty} \chi^{2 n} x^{2 n} ; \quad 4\left[26^{\prime}\right] \\
& \quad(2 n-1) ! !=1.3 .5 \ldots(2 n-1) ; \quad(-1) ! !=1
\end{aligned}
$$

dove

da cui, per il teorema di Cauchy si ha:

$$
\frac{1}{1-\chi^{2} x^{2}} \cdot \frac{1}{\left(1-x^{2}\right)^{1 / 2}}=\sum_{0}^{\infty} p_{n}(\gamma) x^{2 n}
$$

ove si e posto:

$$
p_{n}(\chi)=\sum_{j=0}^{n} \frac{(2 j-1) ! !}{2^{j} j !} \chi^{2 i: \cdots}
$$

Sostituendo la $4[27]$ nel secondo termine della $4[26]$, e considerando che per grandi valori di $a$ si ha:

$$
\int_{0}^{1} x^{-n} e^{-a x} d x \simeq \int_{0}^{\infty} x^{2 n} e^{-i a x} d x=\frac{\sqrt{\pi}(2 n-1)}{2^{n+1}} \frac{1}{(2 a)^{n+1 / 2}}
$$


si perviene allo sviluppo asintotico:

$$
\int_{0}^{1} \frac{e^{-2 a x}}{1-\chi^{2} x^{2}} \frac{d x}{\sqrt{1-x^{2}}} \simeq \sqrt[1]{\pi} \sum_{0}^{\infty} \frac{(2 n-1) ! !}{2^{n+1}} p_{n}(\chi)(2 a)^{-(n+1 / 2)} \cdot 4[30]
$$

Per ottenere un analogo sviluppo del primo integrale osserviamo anzitutto ehe bisogna variare il procedimento, dato che $\lambda$ può essere talmente piccolo da non permettere lo sviluppo in serie di Taylor dell'espressione $\left[1 /\left(\chi^{2}+\lambda^{2}\right)\right]$.

Cambiando la variabile e ponendo:

$$
x=\lambda y, \quad b=\lambda \sqrt{2 a},
$$

si ottiene allora:

$$
\lambda \int_{0}^{1} \frac{e^{-2 a x}}{\lambda^{2}+x^{2}} \cdot \frac{d x}{\sqrt{1-x^{2}}}=\int_{j}^{1 / \lambda} \frac{e^{-b^{2} y^{2}}}{y^{2}+1} \cdot \frac{d y}{y^{2} \sqrt{1-\lambda^{2}}} .
$$

Sviluppando il radicale in serie di potenze:

$$
1 / \sqrt{1-\lambda \cdot y^{2}}=\sum_{0}^{\infty} \frac{(2 n-1) !}{2^{n} \cdot n !} \hat{n}^{n} \cdots \cdot y^{2 n}
$$

si perviene al seguente sviluppo asintotico:

$$
\lambda \int_{0}^{1} \frac{e^{-2 a x}}{\lambda^{2}+x^{2}} \cdot \frac{d x}{\sqrt{1+x^{2}}} \simeq \sum_{0}^{\infty} \frac{(2 n-1) ! !}{2^{n} \cdot n !} \lambda^{2 n} f_{n}(b)
$$

essendo $f_{n}(b)$ dato dall'integrale (si sostituisce di nuovo il limite superiore con $+\infty$, trascurando i termini dell'ordine $e^{-2 a}$ ):

$$
f_{n}(b)=\int_{0}^{\infty}\left(y^{2 n} \cdot e^{-b^{2} y^{2}}\right) /\left(y^{2}+1\right) \cdot d y
$$

Per determinare la $f_{n}(b)$ osserviamo anzitutto che si ha:

$$
\left.\frac{y^{2 n}}{y^{2}+1}=\frac{y^{2 n}-(-1)^{n}}{y^{2}+1}+-1\right)^{n}=(-1)^{n}\left[\frac{1}{y^{*}+1}-\sum_{i=0}^{n-1}(-1)^{1} y^{2 j}\right],
$$

e quindi sostituendo nella 4[33], e tenendo presente la 4[29] (con $b$ = al posto di $2 a$ ) si ottiene:

$$
f_{n}(b)=(-1)^{n}\left[f_{o}(b)-\gamma^{-} \pi \sum_{i=0}^{n-1}(-1)^{j} \frac{(2 j-1) ! !}{2^{j+1} \cdot b^{2 j+1}}\right] .
$$


Rimane da determinare la $f_{0}(b)$. Si ha:

$$
f_{0}(b) e^{-b^{2}}=\int_{0}^{\infty} \frac{e^{-(y-1) b^{2}}}{y^{2}+1} d y
$$

che derivata rispetto $a b$ da:

$$
\frac{\vec{\omega}}{d b}\left[f_{\circ}(b) e^{-b^{2}}\right]=-2 b \int_{0}^{\infty} e^{-b^{2} y^{2}-b^{2}} d y=1 / \pi \cdot e^{-b^{2}},
$$

da cui si ricava:

$$
f_{o}(b)=1^{\prime} \bar{\pi} e^{b^{2}} \int_{b}^{\infty} e^{-x^{2}} d x=1 / \bar{\pi} e^{b^{2}}\left(\frac{1 / \bar{\pi}}{2}-\int_{0}^{b} e^{-x^{2}} d x\right)
$$

quindi la $f_{0}(b)$ si esprime mediante una funzione tabellata (funzione di Gauss).

Raccogliendo i risultati 4[26] - 4[35], si può scrivere la formula asintotica di $u(r, t)$ per grandi valori $d i\left[\beta \mid t^{2}-v^{2} / r^{2}\right]$.

Posto:

$$
\begin{aligned}
& \lambda=\frac{\sqrt{t+r / v}-\sqrt{i-r / v}}{2^{4} \sqrt{t^{2}-r^{2} / v^{2}}}, \\
& \chi=\frac{2 \sqrt[4]{t^{2}-r^{2} / v^{2}}}{\sqrt{t+r / v}+\sqrt{t-r / v}} \\
& b=\prod^{/ \frac{\beta}{2}}(\sqrt{t+r / v}-\sqrt{t-r / v}), \quad d=\sqrt{2 \beta} \cdot \sqrt[4]{t^{2}-r^{2} / v^{2}}, \\
& q_{n}(x)=\frac{(2 n-1) ! !}{2^{n+1}} \sum_{j=0}^{n} \frac{(2 j-1) ! !}{2^{\prime} j !} \chi^{2(n-j)} \\
& g_{n}(b)=(-1)^{n} \frac{(2 n-1) ! !}{2^{n} n !}\left\{g_{0}(b)-\sum_{j=0}^{n-1}(-1)^{j} \frac{(2 j-1) ! !}{2^{j} ! 1} v^{2} ! 1\right\}, \text { per } n \leqslant 1 \\
& g_{0}(b)=e^{b^{2}} \int_{b}^{\infty} e^{-x^{2}} d x=e^{b^{2}}\left(\frac{\sqrt{\pi}}{5}-\int_{0}^{b} e^{-x^{2}} d x\right)
\end{aligned}
$$

si ha per lo sviluppo asintotico di:

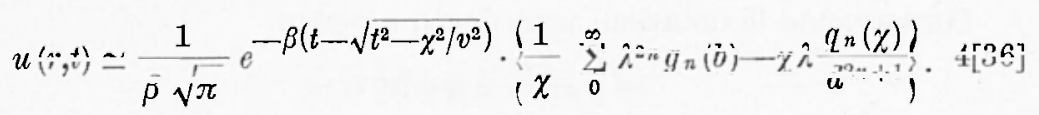


Diamo i primi termini dello sviluppo asintotico. Si ha:

$$
\begin{gathered}
q_{0}=\frac{1}{2}, \quad q_{1}=\frac{1}{4}\left(\frac{1}{2}+\chi^{2}\right) \\
q_{2}=\frac{3}{8}\left(\frac{3}{8}+\frac{1}{2} \chi^{2}+\chi^{3}\right), \quad q_{3}=-\frac{15}{16}\left(\frac{15}{16}+\frac{3}{8} \chi^{2}+\frac{1}{2} \chi^{2}+1\right) \\
g_{1}=\frac{1}{2}\left[\frac{1}{2 b}-g_{0}(b)\right], \quad g_{2}=\frac{3}{8}\left[g_{0}(b)-\frac{1}{2 b}+\frac{1}{4 b^{3}}\right] \\
g_{3}=\frac{5}{16}\left[\frac{1}{2 b}-\frac{1}{4 b^{3}}+\frac{3}{8 b^{5}}-g_{0}(b)\right] .
\end{gathered}
$$

Si osservi inoltre che avendosi per alti valori di $b$ :

$$
g_{\mathrm{o}}(b) \simeq \sum_{0}^{\infty}(-1)^{n} \frac{(2 n-1) ! !}{2^{n+1} b^{2 n+1}}
$$

si puo, per grandi valori di $b$, sostituire la $g_{n}(b)$ coi primi termini dello sviluppo asintotico dato da:

$$
g_{n}(b) \simeq \frac{(2 n-1) ! !}{n !(2 b)^{2 n+1}} \sum_{0}^{\infty}(-1)^{k} \frac{[2(n+k)-1] ! !}{2^{k} b^{2 k}} .
$$

§5. - Completeremo le formule dedotte nel $\S 4$ per il potenziale, per il caso $\beta t \gg 1$, nell'ulteriore ipotesi $r \ll v t$, considerando tempi che oltre ad essere grandi rispetto al "tempo di relassazione ", sono anche grandi (o le distanze sono sufficientemente piccole), rispetto aI tempo di arrivo del segnale. Siccome generalmente il "tempo di relassazione » è dell'ordine:

$$
\left(10^{-8}, 10^{-6} \text { sec. }\right)
$$

mentre per qualche centinaio di metri il tempo d'arrivo del segnale è dell'ordine di $10^{-6}, 10^{-5}$, questo significa che teniamo conto di misure fatte non prima di qualche decimillesimo di secondo dopo l'inizio del segnale per il "potenziale divetto", o dopo la sua fine per il "potenziale lacunare ».

Terremo inoltre conto, almeno per ora, della presenza della superficie del suolo (su cui s'opera), aggiungendo un fattore 2 nel potenziale $\phi$ (come in precedenzil).

Conservando le notazioni precedenti, e posto:

$$
v(r, t)=2 \beta u(r, t)
$$


in maniera che $v(r, t)$ risulti una grandeza adimensionale, si ottengono per il "potenziale diretto" e per il "potenziale lacunare" le:

$\left\{\begin{array}{l}\phi=\frac{J}{2 \pi \sigma r} \cdot v(r, t), \quad \text { per } \frac{r}{v}<t<T+\frac{r}{v}, \text { "potenziale diretto", } \\ \phi=\frac{J}{2 \pi \sigma r}[v(r, t)-v(r, t-T)], \quad \text { per } T+\frac{r}{v}<t, \text {, potenziale lacunare». }\end{array}\right.$

Supporremo, come si è detto:

$$
\text { a) } \beta t \gg 1 ; \quad \text { b) } r / v t \ll 1 \text {. }
$$

Nell'ipotesi a) si e già dedotta, nel $\S 4$, una formula asintotica di cui ora seriviamo i primi termini (cioe i primi 2 termini della prima sommatoria e il primo termine della seconda - dalla formula per la $u(r, t)-)$.

Col significato dei simboli ivi specificato si ha:

$$
v(r, t)=\frac{2}{\sqrt{v}} \frac{1}{\chi}\left(1-\frac{1}{2} \lambda^{2}\right) \int_{b}^{\infty} e^{-x^{2}} d x+\frac{1}{\sqrt{u}} e^{-b^{2}}\left(-\frac{\lambda^{2}}{2 b \chi}-\frac{\chi \lambda}{d}\right) .
$$

Specifichiamo i parametri contenuti nella 5[3] nell'ipotesi $b$ ).

Conserveremo nel primo termine della $5[3]$, termini fino al $2^{\circ}$ ordine, nel $2^{\circ}$ solo quelli di prim'ordine.

Si hanno anzitutto i seguenti sviluppi:

$$
\begin{aligned}
& \sqrt{t+r / v}=\sqrt{t}\left\{1+\frac{1}{2}\left(\frac{r}{v t}\right)-\frac{1}{8}\left(\frac{r}{v t}\right)^{2}+\frac{1}{16}\left(\frac{r}{v t}\right)^{3}-\ldots\right\} \\
& \left.\sqrt{t-r / v}=\sqrt{t}, 1-\frac{1}{2}\left(\frac{r}{v t}\right)-\frac{1}{8}\left(\frac{r}{v t}\right)^{2}-\frac{1}{16}\left(\frac{r}{v t}\right)^{3}-\ldots\right\} \\
& \sqrt{t^{2}-r^{2} / v^{2}}=\sqrt{t}\left\{1-\frac{1}{4}\left(\frac{r}{v t}\right)^{2}+\ldots\right.
\end{aligned}
$$

per grandezze a meno dell'ordine $(r / v t)^{4}$ si ricava:

$$
\chi=1-\frac{1}{8}\left(\frac{r}{v t}\right)^{2}, \quad 1 / \chi=1+\frac{1}{8}\left(\frac{r}{v t}\right)^{2}
$$

a meno dell'ordine $(r / v t)^{3}$ :

$$
\lambda=\frac{1}{2}(r / v t)
$$


da cui, in definitiva a meno di grandezze dell'ordine $(r / v t)^{4}$, si ha:

$$
\frac{1}{\chi}\left(1-\frac{1}{2} \lambda^{2}\right)=1
$$

Per la $b$ ) si ottiene in $2^{\mathbf{a}}$ approssimazione:

$$
b=\sqrt{\frac{\beta}{2 t}} \cdot \frac{r}{v} \cdot\left[1+\frac{1}{8}(v / v t)^{\mathrm{c}}\right] .
$$

Introduciamo la grandezza:

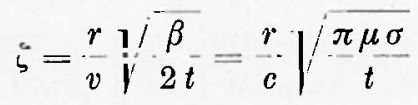

dove se $\sigma$ e misurata in mho/m., e $r$ in metri: $\zeta=\frac{r}{1000} \sqrt{\frac{\pi \mu \sigma}{10 t}}$.

Mediante questa $\zeta$ si ha:

$$
\lambda=\frac{\zeta}{\sqrt{2 \beta t}}, \quad b=\zeta\left[1+\frac{1}{4} \frac{\zeta^{2}}{\beta t}\right] .
$$

Ora nel $2^{\circ}$ termine della 5[3] si può porre inoltre:

$$
b=\zeta, \quad \lambda=1, \quad d=\sqrt{2 \beta t},
$$

per cui esso diventa:

$$
-\frac{1}{\sqrt{\pi}} \cdot \frac{\varrho}{4 \pi t}
$$

e sviluppando in serie di Taylor otteniamo:

$$
\int_{\zeta+\frac{1}{4} \frac{\zeta^{3}}{\beta t}}^{\infty} e^{-x^{2}} d x=\int_{\zeta}^{\infty} e^{-x^{2}} d x-\frac{1}{4} \frac{\zeta^{3}}{b t} \hat{v}^{-\zeta^{3}} .
$$

Introducendo inoltre il "tempo di relassazione"

$$
\theta=\frac{1}{2 \beta}=\frac{\varepsilon}{4 \pi \sigma}, \text { dove } \sigma \text { e dato in } \mathrm{mho} / \mathrm{m}
$$

si ha:

$$
\theta=\frac{\varepsilon \cdot 10^{-11}}{0,36 \pi \sigma}
$$


perveniamo finalmente per la $v(r, t)$ alla seguente espressione:

$$
v(r, t)=1-\frac{2}{\sqrt{\pi}} \int_{0}^{\zeta} e^{-x^{2}} d x-\frac{\theta}{2 t \sqrt{\pi}} \zeta e^{-\zeta^{2}}\left(1+2 \zeta^{2}\right)
$$

Se anche $\zeta$ e abbastanza piccolo, il che praticamente avviene sempre, si puo dare alla $v(r, t)$, a meno del termine dell'ordine $\zeta^{5}$ la forma:

$$
v(r, t)=1-\frac{?}{\sqrt{\pi}}\left(\zeta-\zeta^{3} / 3\right)-0 \zeta / 2 t \sqrt{\pi}
$$

dove nel $2^{\circ}$ termine si e anche trascurato il termine d'ordine superiore $\theta \zeta^{3} / t$.

Si noti che essendo il $6^{\circ}$ termine della $5[8]$ funzione semplicemente di $\zeta$, si ha il risultato che il potenziale sara nelle nostre ipotesi solo in $2^{\text {a }}$ approssimazione dipendente da $\varepsilon$. Infatti $\varepsilon$ entra solo nel tempo 0 di relassazione.

\section{I. - Il potenziale divetto.}

Esso e dato da:

$$
\left.\phi=\frac{J}{2 \pi \sigma r} \mid 1-\frac{\zeta}{\sqrt{\pi}}\left(2-\frac{0}{2 t}\right)+\frac{2}{3 \sqrt{\pi}} \zeta\right] .
$$

Il $1^{\circ}$ termine rappresenta il potenziale d'una corrente stazionaria di intensita $J$; il $2^{\circ}$, essendo $\zeta$ proporzionale ad $r$, una correzione indipendente dalla distanza, il $3^{\circ}$ rappresenta una correzione che cresce col quadrato della distanza.

In misurazioni tipo Wenner (dove viene rilevata solo la d.d.p., differenza di potenziale), il secondo termine non dara contributo, mentre il $3^{\circ}$ dara un contributo talmente piccolo $\left(\zeta\right.$ e dell'ordine $10^{-1}, 10^{-2}$ per distanze di qualche centinaio di metri), da non influire sulle misure.

Il "potenziale diretto" quindi anche per $T$ dell'ordine di qualche decimo di sec. sara dato essenzialmente dal potenziale di ma corrente stazionaria della stessa intensita.

\section{II. - Il "Potenziale lacumare ».}

Esaminiamo il "potenziale lacunare", misurato in un tempo $\tau$ dopo la fine dell'impulso. Si ponga cioè, nella seconda delle 5[2]

ed inoltre

$$
t=T+\tau
$$

$$
\nu=\tau /(\tau+T) \text {. }
$$


Indicando ora con $\zeta$ l'espressione 5[4], dove al posto di $t$ si è messo $\tau$, si ottiene per la $\phi$ :

$$
\phi=\frac{J}{2 \pi \sigma v} \cdot \frac{1}{\sqrt{\pi}} \cdot\left[(1-\sqrt{\nu}) \zeta(2-0 / 2 t)-\frac{2}{3}\left(1-\sqrt{v^{3}}\right) \zeta^{3}\right] \cdot 5[10]
$$

Anche qui il primo termine rappresenta una grandezza costante, che quindi non compare nelle misure di differenza di potenziale. Se si usa ad es. il dispositivo Wenner per eseguire tali misure, con distanza di sonde $a$ e distanza tra gli elettrodi $3 a$ (beninteso che le correnti ai 2 elettrodi si mandino contemporaneamente colla stessa intensita e di segno contrario), il potenziale e dato da:

$$
\varphi=2[\phi(a)-\dot{\varphi}(2 a)]
$$

Utilizzando la $5[10]$ si ha:

$$
\varphi=\frac{J}{2 \pi \sigma l} \frac{1}{\sqrt{\prime} \pi} \cdot\left(1-\sqrt{\nu^{3}}\right) \zeta^{3}
$$

dove con $\zeta$ s'intende la $5[4], r$ e sostituito con $a, t$ con $\tau$. Come s'è già detto $\zeta$ in generale sara del'ordine di $10^{-2}, 10^{-1}$, dipendentemente dall'istante dopo la fine dell'impulso in cui vengono fatte le misure del potenziale, dalla distanza, e dal $\sigma$. Per bassi valori di $\sigma$ detto potenziale scendera anche a $10^{-3}$.

C'e da aspettarsi cioe che se il rapporto $\phi / J$ e dell'ordine Volt/Amp., per il " potenziale diretto ", esso sara dell'ordine del microvolt/Amp. pej" il "potenziale lacunare".

Inoltre dalla 5[12] risulta che detto potenziale nella configurazione Wenner da un contributo positivo crescente con la distanza. Nelle misure d'Evjen questi risultati non sono affatto rispecchiati.

E vero che l'ordine di grandezza del rapporto $\phi / J$ e di qualche microvolts/Amp., e che quindi i dati sarebbero difficili ad interpretarsi come un " potenziale divetto" (cioe un impulso riflesso che arriva con un certo ritardo), essendo il valore di $\phi / J$ del "potenziale diretto" dell'ordine di Volt/Amp. per i dati considerati dall'Autore. Questi tenta d'interpretarli come indice di una costante dielettrica $\varepsilon$ dell'ordine di $10^{8}$. A parte l'inconsistenza fisica di una simile ipotesi, ci sono altre ragioni che l'escludono. Infatti si tratta sostanzialmente di attribuire al suolo un " tempo di relassazione " $\theta$ piuttosto grande. Coi dati di Evjen $\theta$ risulte- 
rebbe dell'ordine di 10 sec., quindi assai superiori al periodo usato. Nella nostra teoria bisognerebbe quindi per la $v(r, t)$ usare le formule del $4^{\circ}$ paragrafo dedotte per piccoli valori di $\beta \sqrt{t-r^{2} / v^{2}}$, cioe quelle che esprimono $I$ mediante le funzioni di Bessel. Ora da queste formule si vede che nell'ambito della loro validita, il "potenziale divetto" risulta dello stesso valore di quello "lacunare".

Il risultato non cambiera in modo essenziale se ad un impulso si sostituisce una successione d'impulsi alternati. Il fenomeno è anche fisicamente evidente: un "tempo di relassazione" di un ordine uguale o superiore alla frequenza degli impulsi, avra come effetto di livellare la corrente, di far sparire cioe qualumque differenza qualitativa tra gli ordini di grandezza della corrente "diretta " e quella "lacunare". Sarebbe stato quindi facile verificare la presenza di un alto "tempo di relassazione» mediante la misura anche del "potenziale diretto". Ora se un fenomeno simile si fosse effettivamente presentato sarebbe stato difficile non notarlo. Infatti una così forte riduzione del "potenziale diretto ", in un rapporto di quasi un milione in confronto col potenziale della corrente stazionaria, sarebbe difficilmente sfuggito all'osservazione. Il disaccordo non può quindi essere spiegato con un così alto valore del "tempo di relassazione" ".

Per spiegare il disaccordo rimane da vedere ancora l'influenza che sul "potenziale lacunare" puo avere la limitazione superficiale e l'atmosfera sovrastante alla superficie del suolo. Infatti si e fatta l'ipotesi (che e soddisfatta nel caso di c.c.) che il problema può essere trattato come se si trattasse d'un mezzo omogeneo che riempie tutto lo spazio, salvo moltiplicare i risultati per 2. C'e da aspettarsi che la correzione sia piccola fino a quando la frequenza si mantenga piuttosto bassa ( $T$ piuttosto grande). Questo significa fisicamente che vengono completamente trascurate eventuali cariche che si formano sulla superficie terrestre. Ora e evidente che per quanto piccola possa essere questa influenza della superficie terrestre sul "potenziale diretto", la cosa si presenta in maniera completamente diversa per quel che riguarda il "potenziale lacunare ». Infatti quest'ultimo e talmente piccolo nella nostra teoria del mezzo omogeneo indefinito, che la correzione provocata dalla presenza d'una superficie sulla quale si possono formare delle cariche superficiali, può dar luogo a delle correzioni piccolissime per quel che riguarda il "potenziale diretto", ma notevolmente grandi in confronto a.l "potenziale lacunare" sopra calcolato. C'è da aspettarsi che buona parte dell'andamento del "potenziale lacunare" sia dato essenzialmente dalla discontinuita della superficie terrestre. Si puo quindi intanto con- 
cludere che per interpretare questi dati e necessario sviluppare una teoria tipo Sommerfeld, che tien conto della superficie terrestre. Solo allora potremmo trarre delle conclusioni attribuendo un eventuale disaccordo rispetto alle inomogeneita del terreno. Questo sara sviluppato nella Parte $\amalg$.

\section{PARTE}

In questa II Parte diamo una teoria del "potenziale geoelettrico" a cominciare da quello generato da un elettrodo alimentato da una "corrente a gradino in suolo omogeneo, tenendo conto dell'effetto della sua superficie, potenziale misurato in decorsi di tempo grandi rispetto al "tempo di relassazione ".

In realta in genere s'adopera una corrente ad andamento rettangolare, ma questa si può considerare quale una sovrapposizione di due correnti a gradino di uguale intensita e di segno contrario, reciprocamente sfasate della durata dell'impulso.

A differenza di quanto svolto nella Parte I terremo particolarmente conto dell'influenza della superficie terrestre sulle grandezze elettriche in oggetto d'esame.

Identificata la superflcie orizzontale del suolo quale piano $(x, y)$, la verticale verso il basso come asse $z$, immagineremo che il flusso di corrente venga fornito mediante un cavo verticale indefinito percorso da una data corrente $I$.

Tutte le grandezze fisiche vengono considerate, come nella Parte I nel sistema elettrostatico C.G.S., permanendo gli stessi simboli.

Com'e noto con correnti paralleli all'asse $z$, e superfici delimitanti i diversi mezzi paralleli al piano $(x, y)$, il c.e. $\vec{E}$ e il c.m. $\vec{H}$, si possono esprimere mediante un potenziale elettrico scalare $(\psi)$ e un potenziale vettore parallelo all'asse $z$ :

$$
\begin{gathered}
\vec{A}\left(A_{x}=0, A_{y}=0, A_{z}=0\right) \\
E_{x}=-\frac{\partial \psi}{\partial x}, \quad E_{y}=-\frac{\partial \psi}{\partial y}, \quad E_{z}=-\frac{\partial \psi}{\partial z}-\frac{\mu}{c} \dot{A} \\
H_{x}=\frac{\partial A}{\partial y}, \quad H_{y}=-\frac{\partial A}{\partial x}, \quad H_{z}=0 .
\end{gathered}
$$


Per di piu $A$ e $\psi$ soddisfano le:

$$
\left\{\begin{array}{l}
\Delta A-\frac{u \varepsilon}{c^{2}} \ddot{A}-\frac{4 \mu \sigma \pi}{c^{2}} \dot{A}=-\frac{4 \pi}{c} J \\
\Delta \varphi-\frac{\mu \varepsilon}{c^{2}} \ddot{\psi}-\frac{4 \mu \sigma \pi}{c^{2}} \dot{\psi}=-\frac{4 \pi}{\varepsilon} \bar{I}
\end{array}\right.
$$

( $J=$ densita di corrente, $\Gamma$ densita di carica), mentre tra $A$ e $\psi$ intercede la relazione:

$$
\frac{\partial A}{\partial z}+\frac{\varrho}{\dot{v}} \dot{\psi}+\frac{4 \pi \sigma}{c} \psi=0
$$

Sulla superficie di separazione di due mezzi, la nota condizione di continuità per le componenti tangenziali delle $\vec{E}$ e $\vec{A}$, si traduce, in virtu delle $6[1]$, nella condizione che su tale superficie le funzioni $\psi$ ed $A$ debbono rimanere continue. Al loro posto conviene spesso introdure una unica $P$ (componente lungo $x$ del vettore di Hertz):

$$
y=-\frac{\partial P}{\mathrm{o} z}, \quad A=c^{-1}(\varepsilon \dot{P}+4 \pi \sigma P) .
$$

Con questa posizione la 6 [3] risulta soddisfatta identicamente, mentre dalle 6[2] discendono per $P$ (nell'ipotesi che per $t=0$ il campo e assente), le:

$$
\begin{cases}\Delta P-\mu \varepsilon c^{-2} \ddot{P}=\left.4 \pi \varepsilon^{-1}\right|_{\dot{0}} ^{t} J d t & (\sigma=0) \\ \Delta P-\mu \varepsilon c^{-2} \ddot{P}=+4 \pi \sigma c^{-2} \dot{P} . & (\sigma \neq 0) .\end{cases}
$$

Le condizioni di continuità tra due mezzi diventano in virti delle $6[4]$ :

$$
\left\{\begin{array}{l}
\left(\frac{\partial P}{\partial z}\right)_{+}=\left(\begin{array}{l}
\partial P^{-} \\
\partial z^{\prime}
\end{array}\right) \\
\varepsilon_{+} \cdot \frac{\partial P_{+}}{\partial t}+4 \pi \sigma_{+} P_{+}=\varepsilon_{-} \cdot \frac{\partial P}{\partial t}+4 \pi \sigma_{-} P_{-}
\end{array}\right.
$$

Applichiamo al nostro caso quest'equazione, ipotizzando che la corrente $I$ sia funzione solo di $t$ (e non di $z$ ), distribuita uniformemente in un cavo sottilissimo circolare di diametro $a \rightarrow 0$.

Di conseguenza introducendo la funzione $\Delta x$, definita dalle:

$$
\Delta x=\left\{\begin{array}{l}
1 \text { per } 0<x<1 \\
0 \text { per } \quad x<1
\end{array}\right.
$$


posto: $r^{2}=x^{2}+y^{2}$, la $J$ diventa:

$$
J=\frac{I(t)}{\pi a^{2}} \Delta\left(\frac{r}{a}\right) .
$$

Poiche il sistema presenta simmetria di rotazione rispetto all'asse $z$, la $P$, dipendera oltre che da $t$, solo da $r$ e $z$, e si perviene alle:

$$
\left\{\begin{array}{l}
r^{-1} \frac{\partial}{\partial r}\left(r \frac{\partial P}{\partial r}\right)+\frac{\partial^{2} P}{\partial z^{2}}-c^{-2} \ddot{P}=-4 a^{-2} \Delta\left(\frac{r}{a}\right) \int_{0}^{t} I(t) d t \\
\text { per } z<0, \text { (atmosfera); } \\
r^{-1} \frac{\partial}{\partial r}\left(r \frac{\partial P}{\partial r}\right)+\frac{\partial^{2} P}{\partial z^{2}}-\varepsilon c^{-2} \ddot{P}=4 \pi \sigma c^{-2} \dot{P} \\
\text { per } z>0, \text { (suolo). }
\end{array}\right.
$$

La $(P)$ deve inoltre obbedire alle condizioni di continuita:

$$
\begin{aligned}
& \left(\frac{\partial P}{\partial z}\right)_{+_{0}}=\left(\frac{\partial P}{\partial z}\right)_{i-0} \\
& \varepsilon\left(\frac{\partial P}{\partial t}\right)_{+0}+4 \pi \sigma(P)_{+0}=\left(\frac{\partial P}{\partial t}\right)_{-0}
\end{aligned}
$$

avendo contrassegnato con $(+0,-0)$ rispettivamente i valori per $z=0$ delle parti positiva e negativa del piano $x y$, alle condizioni iniziali

$$
P=\dot{P}=0, \quad \text { per } t=0
$$

e quelle all'infinito

$$
\left\{\begin{array}{l}
P \rightarrow 0, \text { per } r \rightarrow \infty, \text { e per } z \rightarrow+\infty \\
P \text { limitata per } z \rightarrow-\infty .
\end{array}\right.
$$

Per comodita di scrittura introduciamo nuove variabili

$$
\begin{cases}\varrho-4 \pi \sigma c^{-1} r, & \zeta=4 \pi \sigma c^{-1} z \\ \tau=4 \pi \sigma t & , \quad \alpha-4 \pi \sigma c^{-1} a \\ \pi=4 \pi \sigma c^{-1} P . & \end{cases}
$$

Eseguiti questi cambiamenti, le $6[9], 6[10], 6[11], 6[12]$, diventano:

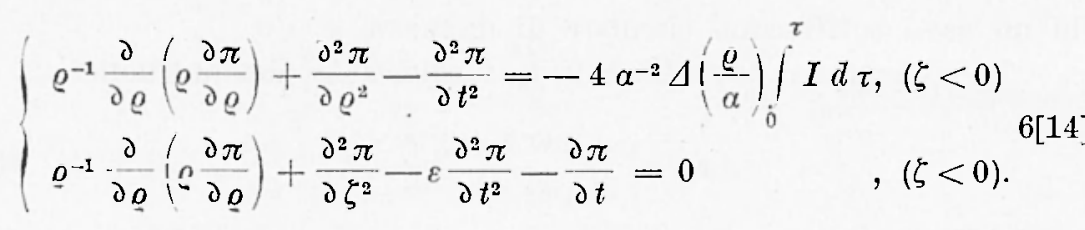


La condizione di contatto comporta:

$$
\left(\frac{\partial \pi}{\partial \zeta}\right)_{+_{0}}=\left(\frac{\partial \pi}{\partial \zeta}\right)_{-0} ; \quad \varepsilon\left(\frac{\partial \pi}{\partial \tau}\right)_{+_{0}}+(\pi)_{+_{0}}=\left(\frac{\partial \pi}{\partial \tau}\right)_{-0} .
$$

Così le condizioni iniziali risultano:

$$
\pi=\frac{\partial \pi}{\partial \tau}=0, \text { per } \tau=0
$$

All'infinito valgono le:

$\pi \rightarrow 0$, per $\varrho \rightarrow+\infty ; \pi=0$, per $\varrho-+\infty ; \pi$ limitata per $\zeta=-\infty .6[17]$

Dovendo in seguito solo interessarei della $\psi$ in superficie $(z=0)$, la indicheremo semplicemente con $\psi$. Dalle 6[4], 6[13], si trae:

$$
\psi=-c^{-1}\left(\frac{\partial \pi}{\partial \dot{\zeta}}\right)_{\zeta=0}
$$

Il nostro problema consiste quindi nel risolvere le $6[14]$, con le condizioni $6[15], 6[16], 6[1 \tau]$ da cui discende $\psi$ tramite la $6[8]$.

Eseguiremo la trasformata di Laplace rispetto al tempo $\tau$, ricordando che:

$$
\bar{F}=\int_{0}^{\infty} e^{-s \tau} F d \tau
$$

Avvalendoci delle condizioni iniziali 6[16], si ricava facilmente con integrazioni per parti:

$$
\int_{0}^{\infty} e^{-\delta \tau} \frac{\partial \pi}{\partial \tau} d \tau=s \bar{\pi} ; \quad \int_{0}^{\infty} e^{-s \tau} \frac{\partial^{2} \pi}{\partial \tau^{2}} d \tau=s^{2} \bar{\pi} .
$$

Analogamente, sempre integrando per parti si lı:

$$
\int_{0}^{\infty} e^{-8 \tau} d \tau \int_{0}^{\tau} I\left(\tau^{\prime}\right) d \tau^{\prime}=s^{-1} \bar{I}
$$

Moltiplicando la $6[14]$ per $e^{-s \tau}$, integrando tra 0 e $\infty$ e tenendo conto delle $6[20]$ e $6[21]$, e posto:

$$
s_{1}^{2}=s(1+\varepsilon s)
$$


la $6[14]$ diventa:

$$
\left\{\begin{array}{ll}
\varrho^{-1} \frac{\lambda}{\partial \varrho}\left(\varrho \frac{\lambda \bar{\pi}}{\partial \varrho}\right)+\frac{\lambda^{2} \bar{\pi}}{\partial \bar{\zeta}^{2}}-s^{2} \bar{\pi}=-4 \alpha^{-2} s^{-1} \Delta(\varrho / \alpha) \bar{I}, & (\text { per } \zeta<0) \\
\varrho^{-1} \frac{\partial}{\partial \varrho}\left(\varrho \frac{\partial \bar{\pi}}{\partial \varrho}\right)+\frac{\partial^{\bar{\pi}} \bar{\pi}}{\lambda \zeta^{2}}-s_{i}^{2} \bar{\pi}=0 & , \text { (per } \zeta<0) .
\end{array} 6[23]\right.
$$

Procedendo analogamente con le $6[15]$ si ottengono le condizioni trasformate di contatto:

$$
\left(\frac{\partial \bar{\pi}}{\partial z z}\right)_{++0}=\left(\frac{\partial \bar{\tau}}{\partial z}\right)_{-0} ; s_{1}^{-}(\bar{\pi})_{+0}=s^{2}(\bar{\pi})_{-0},
$$

mentre le condizioni all'infinito 6[17] permangono le stesse per la $\bar{\pi}$ come per lal $\pi$.

La trasformata della $\psi$ diventa inoltre, in virtu della $6[18]$ :

$$
\bar{\psi}=-c^{-1}\left(\frac{\partial \bar{\pi}}{\partial \zeta}\right)_{\zeta=0} .
$$

Il problema a questo punto consiste nel risolvere il sistema 6[23], con le condizioni 6 [24] e 6[17], e per far cio applichiamo la trasformazione di Fourier-Bessel.

Sia $J_{0}(x)$ la funzione di Bessel d'ordine zero e $P^{*}$ la trasformata di Bessel della $\bar{F}$ :

$$
F^{*}=\int_{0}^{\infty} \varrho J_{0}(\lambda \varrho) \bar{F} d \varrho
$$

Tenendo presente che la $J_{0}(\lambda \varrho)$ è soluzione della

$$
\frac{d}{d \varrho}\left[\varrho \frac{d}{d \varrho} J_{0}(\lambda \varrho)\right]=-\lambda=\varrho J_{0}(\lambda \varrho)
$$

e inoltre soddisfa alla condizione per $\varrho-+\infty$ (si suppone che la $\bar{\pi}$, per $\varrho=+\infty$ si annulli abbastanza fortemente per render nulli i contributi dell'infinito nell'integrazione per parti, e si puo dimostrare che questa condizione e soddisfatta), si ottiene con due integrazioni per parti:

$$
\int_{0}^{\infty} \frac{\partial}{\partial \varrho}\left(\varrho \frac{\partial \bar{\pi}}{\partial \varrho}\right) J_{0}(\lambda \varrho)=-\lambda^{2} \pi^{*}
$$

In piu si ha:

$$
2 \alpha^{-2} \int_{0}^{\infty} \varrho J_{0}(\lambda \varrho) \Delta\left(\begin{array}{l}
\varrho \\
\alpha
\end{array}\right) d \varrho=2 \alpha^{-2} \int_{0}^{\alpha} \varrho J_{0}(\lambda \varrho) d \varrho
$$


che per $\alpha \rightarrow 0$ (avendosi $J(0)=1$ ) tende al valore:

$$
2 \alpha^{-=} \int_{0}^{\alpha} \varrho d \varrho=1
$$

Si moltiplichino le $6[23]$ per $2 J_{0}(\lambda \varrho)$, e si integri tra 0 e $\infty$ : utilizzando le $6[33]$ e $6[34]$, si dedueono per $\alpha \rightarrow 0$ e per $a \rightarrow 0$, le seguenti equazioni differenziali ordinarie:

$$
\begin{cases}\vec{a} \pi^{*} & \left(\lambda^{2}+s^{2}\right) \pi^{*}=-2 s^{-1} I, \text { per } \zeta<0 \\ d \xi^{2} & \text {, per } \zeta<0 . \\ \frac{d^{2} \pi^{*}}{d \zeta^{2}}-\left(\lambda^{2}+s_{1}^{2}\right) \pi^{*}=0 & \text {. }\end{cases}
$$

Bisogna integrare questo sistema con le condizioni che si ottengono trasformando quelle di contatto, e per $z= \pm \infty$, conservanti la stessa forma come per le $\bar{\pi}$.

Esse sono quindi:

$$
\left(\frac{d \pi^{*}}{d \zeta}\right)_{+0}=\left(\frac{d \pi^{*}}{d \zeta}\right)_{-0}, \quad s_{1}^{2}\left(\pi^{*}\right)_{+_{0}}=s^{2}\left(\pi^{* *}\right)_{-0}
$$

e:

$$
\pi^{*} \rightarrow 0, \text { per } \zeta \rightarrow \infty ; \quad \pi * \text { limitata, per } \zeta=-\infty . \quad 6[37]
$$

L'integrazione della 6[35] con le condizioni $6[36]$ e $6[37]$ e del tutto elementare. La soluzione generale che soddisfa le $6[35], 6[37]$, e la prima della $6[36]$ e data dalle:

$$
\begin{cases}\pi^{*}=2 \bar{I}\left(\lambda^{2}+s^{2}\right)^{-1 / 2}\left[-B e^{\zeta \sqrt{\lambda^{2}+s^{2}}}+s^{-1} \cdot\left(\lambda^{2}+s^{2}\right)^{-1 / 2},\right. & \text { per } \zeta<0 \\ \pi^{*}=2 \bar{I}\left(\lambda^{2}+s_{1}^{2}\right)^{-1 / 2} D_{0} \zeta \sqrt{ } \mu^{2}+s_{1}^{*} & \text {, per } \zeta<0 .\end{cases}
$$

La costante $B$ (funzione di $\lambda$ e $s$ ) si determina dalla seconda delle $6[36]:$

da cui:

$$
B\left(\frac{s^{2}}{\sqrt{\lambda^{2}+s_{1}^{2}}}+-\frac{s^{2}}{\sqrt{\hat{\lambda}^{2}+s^{2}}}\right)=\frac{1}{\lambda^{2}+s^{2}}
$$

$$
B=\frac{s \sqrt{\lambda^{2}+o_{1}^{2}}}{\sqrt{\lambda^{2}+s^{2}}}\left[s^{2} \sqrt{\lambda^{2}+s^{2}}+s^{2} \sqrt{\lambda^{2}+s_{1}^{2}}\right]^{-1} .
$$

Come gia detto, noi siamo interessati esclusivamente alla $\psi$, quindi ai preme solo $\psi^{*}$, che si ottiene dalla $6[30]$, trasformandola:

$$
\psi^{*}=-c^{-1}\left(\frac{\partial \pi^{*}}{\partial \zeta}\right)_{\zeta=0}
$$


Dalle 6[41] e 6[38] si ha infatti:

$$
\psi^{*}=\frac{2 \bar{I}}{c} B
$$

con $B$ data dalla $6[40]$.

Una volta cosi determinata $\psi^{*}$, dobbiamo risalire alla $\psi$ invertendo le due trasformazioni, e cominciando con l'inversione di quella di Fourier Bessel. Questa trasformazione inversa si presenta:

$$
\bar{\psi}=\int_{0}^{\infty} \lambda J_{0}(\lambda \varrho) \psi^{*} d \lambda
$$

$o$, in virtu della $6[12]$

$$
\bar{\psi}=2 \bar{I} o^{-1} \int_{0}^{\infty} \lambda J_{0}(\lambda \varrho) B(\lambda) d \lambda .
$$

La trasformata di $B(\lambda)$ si riesce ad esprimerla mediante fumzioni che non coinvolgono la funzione di Bessel. A questo scopo bisogna anzitutto scrivere in maniera diversa la $B$, spezzandola in termini che contengono solo il tipo di ridicali che compaiono nella 6[40]. Con un calcolo un po' laborioso si trova per $B$ :

$$
\begin{aligned}
& B=\frac{1}{s \sqrt{\lambda^{2}+s^{2}}}+\frac{s_{1}^{2}}{s\left(s_{1}^{2}-s^{2}\right)\left(s_{\mathrm{I}}^{2}+s^{2}\right)}\left\lceil\frac{s^{2}}{\sqrt{\lambda^{2}+s_{1}^{2}}+\frac{s_{1}^{2}}{\sqrt{s_{1}^{2}+s^{2}}}}-\right. \\
& \frac{s^{2}}{\left.\sqrt{\lambda^{2}+s^{2}}+\frac{s^{2}}{\sqrt{s^{2}+s_{1}^{2}}}\right]}
\end{aligned}
$$

da cui si puo verificare elementarmente che essa coincide con la 6[40]. Dalla $6[45]$ che da la $B$ si vede subito che il calcolo di $\bar{\psi}$ mediante la 6[44] si riduce a quello d'integrali del tipo:

$$
L=\int_{0}^{\infty} \frac{\lambda d \lambda}{b+\sqrt{a^{2}+\lambda^{2}}} J_{0}(\lambda \varrho),
$$

che si possono trasformare in altri che non contengono piu $J_{0}(\lambda \varrho)$, partendo da una nota relazione di Sommerfeld:

$$
\int_{0}^{\infty} \frac{\lambda d \lambda}{\sqrt{\lambda^{2}+a^{2}}} e^{-c \sqrt{c^{2}+\lambda^{2}}} \cdot J_{v}(\lambda \varrho) d \lambda=\frac{e^{-a \sqrt{\varrho^{2}+c^{2}}}}{\sqrt{\varrho^{2}+c^{2}}} .
$$


Posto:

$$
L(c)=\int_{0}^{\sim} \frac{\lambda d \lambda}{b+\sqrt{a^{2}+\lambda^{2}}} e^{-b c-\sqrt{\lambda^{2}+a^{2}}} \cdot J_{0}(\lambda . o)
$$

si ha:

$$
L(\infty)=0, \quad L(0)=L .
$$

Derivando le $6[47]$ e $6[48]$ rispetto a $c$, e confrontando i due risultati, si ottiene:

$$
\frac{d}{d c} L(c)=e^{-b c} \frac{d}{d c}\left(\frac{e^{-a \sqrt{Q^{2}+c^{2}}}}{\sqrt{\varrho^{2}+c^{2}}}\right)
$$

Integrando la 6[49] rispetto a $c$, tra 0 e $\infty$ e cambiando di segno, ne deriva la:

$$
\begin{aligned}
& I_{1}=-\int_{0}^{\infty} e^{-b c} \frac{d}{d c}\left(\frac{e^{-a \sqrt{o^{2}+c^{2}}}}{\sqrt{\varrho^{2}+c^{2}}}\right) d c= \\
& =\frac{e^{-a o}}{\varrho}-b \int_{0}^{\sim} \frac{d c}{\sqrt{\varrho^{2}+c^{2}}} e^{-b c-a \sqrt{\rho^{2}+c^{2}}} \text {. }
\end{aligned}
$$

Sostituiamo la variabile $c$ nella $x$. mediante la:

$$
x=\left(b c+a \sqrt{\varrho^{2}+c^{2}}\right) / \varrho \sqrt{a^{2}-b^{2}} .
$$

Essendo:

$$
\frac{d x}{\sqrt{x^{2}-1}}-\frac{d c}{\sqrt{\varrho^{2}+c}}
$$

si perviene in virtu delle $6[46], 6[52]$, e $6[51]$ alla:

$$
\int_{0}^{\infty} \frac{\lambda d \lambda}{b+\sqrt{\lambda^{2}+a^{2}}} J_{0}(\lambda \varrho) d \lambda=\frac{e^{-a \varrho}}{\varrho}-b \int_{a / \sqrt{a^{2}-b^{2}}}^{\infty} e^{-x \varrho \sqrt{a^{2}+b^{2}}} \frac{d x}{\sqrt{x^{2}-1}} \cdot 6[53]
$$

Per calcolare la $\bar{\psi}$, basta ora sostituire la $6[45]$ nella $6[44]$, tenendo presente la 6[53], si giunge così alla:

$$
\begin{gathered}
\bar{\psi}=\frac{2 \tilde{I}}{c \varrho}\left(\frac{-s^{3} e^{-s \varrho}}{\left(s_{1}^{2}-s^{2}\right)\left(s_{1}^{2}+s^{2}\right)}+\frac{s s_{1}^{2} e^{-s_{1} \varrho}}{\left(s_{1}^{2}-s^{2}\right)\left(s_{1}^{2}+s^{2}\right)}+\right. \\
\left.+\frac{s^{2} s_{1}^{4} \varrho}{\left(s_{1}^{2}-s^{2}\right) \sqrt{\left(s_{1}^{2}+s^{2}\right)^{3}}} \int_{s_{1}^{-1} \sqrt{s_{1}^{2}+s^{2}}}^{-x \varrho s s_{1} / \sqrt{s_{1}^{2}-s^{2}}}\left(\frac{d x}{\sqrt{x^{2}-1}}\right)\right\}
\end{gathered}
$$


dove:

$$
s_{1}^{2}=s(1+\varepsilon s)
$$

Per ricavare $\psi$ bisogna invertire la trasformata $L$, esplicitando anzitutto la $I(s)$, che finora non ci serviva. Come gia detto noi ci occupiamo della teoria del "gradino potenziale ", quindi per $I(t)$, si ha:

$$
I(t)=0, \quad \text { per } t<0 ; \quad I(t)=I, \text { per } t<0,
$$

da. cui:

$$
I=I / s, \quad \text { dove } I(s)=\int_{0}^{\infty} e^{-s \tau} I d \tau
$$

Essendo l'antitrasformata data mediante l'integrale nel campo complesso:

$$
\psi=\frac{1}{2 \pi \imath} \mid \bar{\psi} e^{s \tau} d s
$$

con $L$ retta parallela all'asse immaginario a destra dell'origine o per un cammino equivalente, si ottiene per $\psi$ la:

$$
\psi=2 I u / c \varrho-I u / 2 \pi \sigma r
$$

con:

$$
u=\frac{1}{2 \pi i} \int_{i_{1}} \frac{d s}{s} g(s) e^{s \tau}
$$

e $g(s)$ è il termine tra parentesi nella $6[5 \pm]$.

E fin qui tutti i calcoli sono stati eseguiti in maniera esatta. D'ora in poi supporremo $\tau \gg 1$, un "tempo d'osservazione " molto grande rispetto al " tempo di relassazione ". Com'è noto dalla teoria delle approssimazioni asintotiche, in questo caso il comportamento dell'integrale $6[59]$, è dato essenzialmente da quello della $g(s) / s$, in quel punto singolare che si trova piu a destra nel piano complesso. Questo punto singolare e il punto $s=0$ : in altre parole si puo sostituire $g(s)$ col suo sviluppo nell'origine, il che corrisponde al fatto fisico che per grandi valori di $\tau$ influiscono essenzialmente le basse frequenze.

Per ricavare quindi la parte principale di $\bar{\psi}$, si puo anzitutto tralasciare il primo termine della $g$, [v. 6[59]], che è regolare per $s=0$, e negli altri sostituire:

$$
s_{1} \operatorname{con} \sqrt{s,} \quad s_{1}^{2}-s^{2} \text { e } s_{1}^{2}+s^{2} \text { con } s .
$$


Porremo cioe nella 6[59]:

$$
g(s)-e^{-\varrho \sqrt{s}}+\varrho \sqrt{s} \int_{1}^{1 / 1 \bar{s}} e^{-x \varrho s} d x / \sqrt{x^{2}-1}
$$

(il radicale indica naturalmente la determinazione con la parte reale positiva).

Si puo dimostrare che tutto cio e giustificato a patto che oltre alla $\tau \gg 1, \varrho$ non sia troppo grande, in maniera che o rimanga molto piccolo rispetto a $\tau^{3 / 2}$.

Questa seconda condizione puo essere espressa anche nella seguente maniera. Posto

$$
\omega=\varrho / 2 \sqrt{r}=\frac{r}{c} \sqrt{\pi \sigma / t}
$$

le condizioni di validita della 6[60] sono:

a) $\tau$ molto grande rispetto all'unita: $\tau \gg 1$

b) $\omega$ non troppo grande rispetto all'unita: $\tau \geqslant 1$.

La $b$ ) in pratica e sempre soddisfatta mentre, in generale in questi casi, $\omega$ appare piuttosto piccola.

Passiamo ora alla valutazione della 6[59], utilizzando la 6[60] per la $g(s)$.

Si osservi che nelle nostre ipotesi il cammino $L$ si puo deformare verso sinistra all'infinito, arbitrariamente. Lo sostituiremo con parabole col vertice sull'asse reale positivo, o nell'origine.

Vediamo anzitutto il primo termine nella 6[60]: il suo contributo alla $(u)$ sarà dato da:

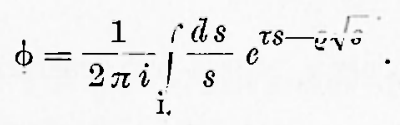

Prendendo per cammino d'integrazione la parabola:

$$
s=\frac{\varrho^{2}}{4 \tau^{2}}(1+i z)^{2}, \quad \text { con }-\infty<z<\infty,
$$

esso diventa:

$$
\phi=\frac{1}{\pi} \int_{-\infty}^{+\infty} \frac{d z}{i z+1} e^{-\omega^{2}\left(1+z^{2}\right)}=\frac{2}{\pi} \int_{0}^{\infty} \frac{d z}{1+z^{2}} e^{-\omega^{2}\left(1+z^{2}\right)} .
$$

Derivando la $6[64]$ rispetto a $\omega$ :

$$
\frac{d \phi}{d \omega}=-\frac{4 \omega}{\pi} \int_{0}^{\infty} e^{-\omega^{2}\left(1+z^{2}\right)} \cdot d z=-\frac{2}{\sqrt{j} \pi} e^{-\omega^{2}}
$$


in virtu della nota formula si ottiene:

$$
\omega \int_{0}^{\infty} e^{-\omega^{2} z^{2}} d z=\int_{0}^{\infty} e^{-x^{2}} d x=\sqrt{\pi} / 2, \quad(z=x / \omega) .
$$

Dalla 6[65], insieme con la $\phi=0$ per $\omega=\infty$, si ha integrando tra $\omega$ e $\infty$, la:

$$
\phi=\frac{2}{v i=} \int_{\omega}^{\infty} e^{-\infty} d z=1-\frac{2}{\sqrt{\pi}} \cdot \int_{0}^{\infty} e^{\tilde{z}^{2}} d z,
$$

termine che e gia stato da noi ricavato nella Parte $I$, quando non si teneva conto dell'influenza della superficie terrestre. Il secondo termine rappresenta l'infiuenza della superficie, e la sua valutazione si presenta in maniera molto piu laboriosa.

Dalle $6[59]$ e $6[40]$ e dato da:

$$
\phi_{1}=\frac{\varrho}{2 \pi i} \int_{i} \frac{d s}{\sqrt{s}} \int_{i}^{1 / i^{-}} e^{-x \varrho s+\tau s} d x / \sqrt{x^{2}-1} .
$$

Scelto come cammino d'integrazione la parabola:

$$
s=-z^{2} \operatorname{con}(-\infty<z<\infty), \quad \sqrt{s}=i u,
$$

la $6[67]$ assume la forma:

$$
\phi_{1}=\frac{\varrho}{\pi} \int_{-\infty}^{+\infty} d z \int_{1}^{-1 / z} e^{-x Q z^{2}-\tau z^{2}} d x / \sqrt{x^{2}-1} .
$$

L'integrale interno per $z>0$ si puo trasformare in $\int_{i}^{-i / z} \frac{e^{x \varrho z^{2}} d x}{\sqrt{x^{2}-1}}=-i \int_{0}^{1} e^{x \varrho z^{2}} d x / \sqrt{1-x^{2}}+\int_{0}^{i / z} e^{-i x \varrho z^{2}} d x / \sqrt{1+x^{2}}, 6[70]$ e analogamente:

$$
\begin{aligned}
& \int_{1}^{-i / z} \frac{d x}{\sqrt{x^{2}-1}} e^{x \varrho z^{2}}=-i \int_{1}^{-i / z} \frac{d x}{\sqrt{1-x^{2}}} e^{x \varrho z^{2}}=i \int_{0}^{1} \frac{d x}{\sqrt{1-x^{2}}} e^{x \varrho z^{2}}- \\
& -i \int_{0}^{-i / z} \frac{d x}{\sqrt{1-x^{2}}} e^{x \varrho z^{2}}=i \int_{0}^{1} \frac{d x}{\sqrt{1-x^{2}}} e^{x \varrho z^{2}}+\int_{0}^{-1 / z} \frac{d x}{\sqrt{1+x^{2}}} e^{i x \varrho z^{2}}
\end{aligned}
$$

per $u<0$. 
Sostituendo quest'espressione nella $6[69]$, e ricordando che:

$$
\int_{-\infty}^{+\infty} f(z) d z=\int_{0}^{\infty}[f(z)+f(-z)] d z
$$

si perviene alla:

$$
\phi_{1}=\left.\frac{\varrho}{\pi}\right|_{0} ^{\infty} d z e^{-\tau z^{2}} \int_{0}^{1 / z}\left(e^{i n x z^{2}}-e^{-i \varrho x z^{2}}\right) \frac{d x}{\sqrt{1+x^{2}}} .
$$

Eseguiamo il seguente cambiamento di variabili nell'integrale 6[71]

Poichè si ha:

$$
x=\xi^{-1}, z=\xi_{\eta} .
$$

la $6[71]$ diventa:

$$
d x d z=\xi^{-1} d \xi d \eta
$$

$$
\phi_{\perp}=\frac{\varrho}{\pi} \int_{-\infty}^{-\infty} \frac{d \xi}{v^{\prime} \xi^{2}+1} \int_{0}^{1} e^{-\eta^{2}\left(\tau \xi^{2}+i \varrho \xi\right)} \cdot d \eta
$$

Se nella 6[73] poniamo:

$$
\xi=z+i_{Q} / 2 \tau=z+1 \omega / \sqrt{\tau}
$$

essa (dopo lo spostamento della retta d'integrazione sull'asse reale, il che e permesso essendo $\omega / \sqrt{\tau}$ piuttosto piccolo), diviene:

$$
\phi_{1}=\frac{\varrho}{\pi} \int_{-\infty}^{+\infty}\left[1+(z+i \omega / \sqrt{\tau})^{2}\right]^{-1 / 2} \int_{0}^{1} d \eta e^{-\left(\tau z^{2}+\omega\right) \eta^{2}} .
$$

Il contributo della $i \omega \sqrt{\tau}$ e dell'ordine di quelli gia trascurati, perciò possiamo sostituire la $6[75]$ con la:

$$
\phi_{1}=\frac{2}{\pi} \int_{-\infty}^{+\infty}\left(z^{-}+1\right)^{1 / 2} d z \int_{0}^{1} d \eta e^{-\eta^{2}\left(\tau z^{2}+\omega^{2}\right)} .
$$

Se indichiamo con $\varphi(x)$ la funzione di Gauss:

$$
\varphi(x)=\int_{0} e^{-y^{2}} d y
$$

cambiando $\eta$ in $\eta=y /\left(\tau^{2} z^{2}+\left(t^{2}\right)^{1 / 2}\right.$ a ntilizzando la $\left.6[\tau]\right]$, si ha:

$$
\phi_{1}=\frac{2 \varrho}{\pi} \int_{0}^{\infty} \varphi\left(\sqrt{\omega^{2}+\tau^{2} z^{2}}\right) d z / \sqrt{z^{2}+1} \cdot \sqrt{\tau z^{2}+\omega^{2}} .
$$


Ponendo:

$$
x=\left(\tau^{2} z^{2}+\omega^{2}\right)^{1 / 2}, \quad z=\frac{1}{\sqrt{\tau}}\left(x^{2}-\omega^{2}\right)^{1 / 2},
$$

la 6 [78] diventa:

$$
\phi_{1}=\frac{4 \omega}{\pi} \int_{\omega}^{\infty} \varphi(x) d x / \sqrt{x^{2}-\omega^{2}} \mid 1+\frac{x^{2}-\omega^{2}}{\tau} .
$$

D'altronde si ha:

$$
\varphi(x)=\frac{\sqrt{x}}{2}-\int_{x}^{\infty} e^{-y^{2}} d y
$$

il che permette di decomporre $\phi_{1}$ nella differenza:

La $\phi^{\prime}$ e data da:

$$
\phi_{1}=\phi^{\prime}-\phi^{\prime \prime} \text {. }
$$

$$
\phi^{\prime}=\frac{2 \omega}{\sqrt{\pi}} \int_{\omega}^{\infty} d x \cdot\left(x^{2}-\omega^{2}\right)^{-1 / 2}\left(1+\frac{x^{2}-\omega^{2}}{\tau}\right)^{-1 / 2}
$$

Posto:

$$
x-=\frac{\omega}{\xi},
$$

la $6[83]$ si serive:

$$
\phi^{\prime}-\frac{2 \omega}{v \pi} \int_{0}^{1} d \xi \cdot\left(1-\xi^{2,-1 / 2}\left[\xi^{2}\left(1-\frac{\omega^{2}}{\tau}\right)+\frac{\omega^{2}}{\tau}\right]^{-1 / 2}\right.
$$

che con l'ulteriore cambiamento di variabile:

$$
\xi=\cos \varphi
$$

si trasforma nell'integrale ellittico completo di $1^{\text {a }}$ specie $K$ :

$$
\phi^{\prime}=\frac{2 \omega}{\sqrt{\pi}} \int_{0}^{\pi / 2} d \varphi\left|1-\left(1-\frac{\omega^{2}}{\tau}\right) \operatorname{sen}^{2} \varphi\right|^{-1 / 2}=\pi\left(\mathbf{i} 1-\frac{\omega^{2}}{\tau}\right) \cdot 6\left[85^{\prime}\right]
$$

Ora dato che $\omega^{2} / \tau$ e piccolo si ha, a meno di termini dell'ordine di quello gria trascurato e in virti della nota formula:

$$
K\left(\sqrt{1-K-)} \sim \lg \frac{4}{K} \text {, per } K\right. \text { molto piecolo }
$$

Ia:

$$
\phi^{\prime}=\frac{\omega}{\sqrt{\pi}}[4 \lg 2-\lg \tau+2 \lg \omega]
$$


Per ricavare la $\oint^{\prime \prime}$ si puo trascurare nellat radice del denominatore il termine

$$
\frac{x^{2}-\omega^{2}}{\tau}
$$

che comporta solo termini dell'ordine gia trascurato. Con cio essa diventa:

$$
\phi^{\prime \prime}=-\frac{ \pm(\omega)}{\pi} \int_{\omega}^{\infty} d x\left(x^{2}-\omega^{2}\right)^{-1 / 2} \int_{\omega}^{\infty} e^{-y^{2}} d y
$$

o posto:

$$
\begin{gathered}
x=\xi \omega, \\
\phi^{\prime \prime}=\left.\frac{4 \omega}{\pi}\right|_{i} ^{\infty} d \xi\left(\xi^{2}-1\right)^{-1 / 2} \int_{\omega_{5}^{5}}^{\infty} e^{-y^{n}} d y .
\end{gathered}
$$

Indichiamo ora con $h(\omega)$ l'espressione:

$$
h((1))=\frac{4}{\pi} \int_{i}^{\infty}\left(\xi^{2}-1\right)^{-1 / 2} d \xi \int_{\omega \xi}^{\infty} e^{-y^{2}} d y .
$$

Derivando lispetto a $\omega$ la $6[91]$ si hal:

$$
\frac{d h}{d \omega}=-\frac{4}{\pi} \int_{\mathrm{i}}^{\infty}\left(\xi^{2}-1\right)^{1 / 2} \cdot 53 \xi \cdot s^{-\omega^{2} \xi^{2}},
$$

posto:

$$
\xi=\left(1+\eta^{2} / \omega^{2}\right)^{1 / 2}
$$

essa diventa:

$$
\frac{d h}{a \omega}=-\frac{ \pm}{\pi \omega} e^{-\omega^{2}} \int_{0}^{\infty} e^{-\eta^{2}}=-\frac{2}{\sqrt{\pi}} \frac{e^{\omega^{2}}}{\omega} .
$$

Dalla 6[94] integrandola tra $\omega$ e $\infty$, poiche in piu $h(\infty)=0$, si ottiene:

$$
h(\omega)=\left.\frac{2}{\sqrt{\pi} \bar{\tau}}\right|_{\omega} ^{\infty} \frac{e^{-u^{2}}}{u} d u
$$

Posto ancor"a:

$$
u=\sqrt{x}
$$

per $\phi^{\prime \prime}=\omega h(\omega)$, si ha l'espressione:

$$
\phi^{\prime \prime}=\frac{\omega}{\sqrt{\pi}} \int_{i^{2}}^{\infty} \frac{e^{-x}}{x} d x
$$


$A$ questo punto possiamo racogliere i risultati delle $6[66], 6[87]$, $6[97]$ e inserirle nella $6[58]$.

Per

$$
\omega=\frac{r}{\mathrm{c}} \sqrt{\frac{\pi \sigma}{t}}, \tau= \pm \pi \sigma t
$$

per il potenziale al tempo $t$ e alla aistanza $r-c o n ~ r, t$ tali per cui $\tau \gg 1$ ew non troppo grande rispetto all'unita - si ha l'espressione asintotica:

$$
\begin{gathered}
\psi=\psi_{0}+\psi_{1} \\
\psi_{0}=I / 2 \pi \sigma r \\
\psi_{1}=[f(\omega)+\lg \tau] \cdot I / c \sqrt{\pi \tau} \\
f(\omega)=4 \lg 2-2 \lg \omega-\int_{\omega^{2}}^{\infty} d x \cdot \frac{e^{-x}}{x}-2 \int_{\omega}^{\infty} e^{-x^{2}} d x,
\end{gathered}
$$

con due integrali funzioni ben note e tabulate. Dai loro sviluppi in serie deriva quello della $f(\omega)$ :

$$
f(\omega)=4 \lg 2-2+\gamma-\sum_{1}^{n}(-1) J^{n-1} \omega^{1 n} / n(2 n+1) n !
$$

(con $\gamma=0,57721$ costante d'Eulero-Mascheroni).

Se le grandezze sono misurate $\tau$ in metri, $\sigma$ in ohm.metri-1, $J$ in Ampere, $\psi$ in Volt, le [A] si scrivono:

$$
\omega=\frac{r}{1000} \sqrt{\pi \sigma / 10 t} \quad, \quad \tau=3.6 \pi 10^{10} \sigma t
$$

le $[\mathrm{B}],[\mathrm{C}]$, e naturalmente anche le $[\mathrm{E}]$ e $[\mathrm{F}]$ rimangono invariate, mentre per la [D] si ha:

$$
\psi_{1}=\frac{I}{2000 \pi \sqrt{10^{8} t}}[f(\omega)+\lg \tau]=\frac{3 n I}{\sqrt{\pi t}}[f(\omega)+\lg \tau] .
$$

Per quel che riguarda l'ordine di grandezza dei termini trascurati, essi sono di due tipi:

1) termini in $\varepsilon / \tau, \theta / t, \operatorname{con} \theta$ " tempo di relassazione ". Si può dimostrare che questi termini riguardano esclusivamente $\psi$, nel senso che essi presentano una struttura simile a $\psi_{1}$, con coefficienti diminuiti nel rapporto 0/t. Essendo $\theta / t$ nella nostra teoria estremamente piccolo, essi sono trascurabili rispetto a $\psi_{1}$. Questo fatto $\dot{e}$ importante, in quanto essendo in pratica la $\psi_{1}$ molto piccola rispetto a $\psi_{0}$, poteva sorgere il dubbio che l'eventuale presenza di termini dell'ordine $\psi_{0} \theta / t$ contribuisse ai termini dell'ordine di grandezza di $\psi_{1}$. 
2) termini dell'ordine di grandezza $\psi_{0} e^{+/ s}:$ questi nonostante che $\psi_{0}$ sia di solito molto piu grande di $\psi_{1}$, risultano anch'essi completamente trascurabili rispetto a $w_{1}$. dato il fattore esponenziale.

Bisogna ancora aggiungere che dalla struttura della $f(\omega)$ appare che essa tende all' $\infty$, se pur lentamente, quando $\omega$ (cioe $r$ ) cresce all' $\infty$, il che parrebbe un assurdo, dovenclo il potenziale annullarsi all' $\infty$. Questa contraddizione è pero apparente, perchè la $6[10]$ è valida solo per valori di $\omega$ non troppo grandi. Allorquando $\omega$ diventa dell'ordine di $\sqrt{\tau}$ (cioe $\tau$ dell'ordine di $c t$, cosa che in pratica non interessa), la [D] cessa di essere valida.

Per concludere aggiungiamo ancora qualche osservazione "sull'influenza della superficie del suolo".

Se confrontiamo la $\psi_{1}$ calcolata con la [D], con l'analoga espressione da noi dedotta nella Parte I (trascurando l'effetto della superficie), si vecle che all'integrale:

$$
-\frac{2}{\omega} \int_{0}^{\omega} e^{-x^{2}} d x
$$

si sostituisce ora $f(\omega) \cdots \lg \tau$.

Dalla [E] appare che il contributo dei primi tre termini, che rappresentano l'effetto superficiale, è tutt'altro che trascumbile rispetto al contributo del quarto termine che rappresenta la teoria senza tenere conto dell'effetto superficiale (la qual cosa e ancora piu evidente scrivendo gli sviluppi in serie).

Siccome le misure si riferiscono sempre a differenze di potenziale, interessa solo la:

$$
[f(\omega)-f(0)]
$$

che, per la [F], è data dallo sviluppo:

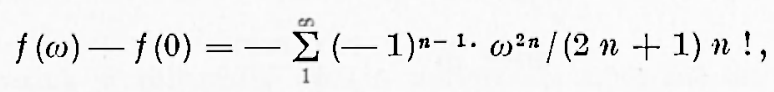

mentre la corrispondente espressione per:

$$
-\frac{2}{\omega} \int_{0}^{\omega} e^{-x^{2}} d x
$$

e data da:

$$
-\frac{2}{(1)} \int_{0}^{\omega} e^{-x^{2}} d x+2=\sum_{1}^{\infty} 2(-1)^{n-1} \omega^{2 n} /(2 n+1) n !
$$


La espressione 6[98] e non solo diversa dalla 6[99] ma ne differisee addirittura nel segno.

Il contributo della superficie è quindi piu importante del termine calcolato in sua assenza, in quanto la sua aggiunta cambia il segno alla differenza:

$$
\psi_{1}(r, t)-\psi_{1}(0, t)
$$

Da queste considerazioni deriva che la teoria di Horton, per quel che riguarda una corrente a gradino, non e corretta (come non lo sono le altre finora apparse su quest'argomento), in quanto si trascura l'effetto superficiale. La funzione $\psi_{1}$ (che coincide con quella dedotta nella Parte I, a meno del termine in $\theta / t$ ), non e piu precisa di quanto non lo sia la semplice formula:

$$
\psi=\psi_{0}=I / 2 \pi \sigma r
$$

(valida per una corrente continua).

Risultando le formule indipendenti da $\varepsilon$, si puo concludere che, in un suolo omogeneo, una corrente a gradino puó far sentire l'effetto della costante dielettrica $\varepsilon$ solo per tempi e distanze che non soddisfino alle condizioni $A, B, C, D, E, F$, cioe per tempi che diventano confrontabili col "tempo di relassazione" o col tempo d'arrivo del segnale.

§ 7. - Abbiamo cosi calcolato il potenziale dovuto ad una corrente a gradino:

$$
\psi=\frac{I}{2 \pi \sigma r}+\frac{I}{2 \pi c \sqrt{\sigma t}}( \pm \lg 2-2+\gamma+\lg \tau)-\frac{I}{2 \pi c \sqrt{\sigma t}} f(\omega) \quad 7[1]
$$

da cui:

$$
\begin{gathered}
f(\omega)=2 \lg (\omega)+\int_{\omega^{2}}^{\infty} \frac{e^{-x}}{x} d x+\frac{2}{\omega \int_{0}^{p} e^{-x^{2}}} d x+\gamma-2 ; \\
\text { dove } \omega=\frac{r}{c} \sqrt{\frac{\pi \sigma}{t}} .
\end{gathered}
$$

La $f(\omega)$ si puo scrivere, sviluppandola in serie di Taylor:

$$
f(\omega)=\sum_{1}^{\infty} \frac{(-1)^{n-1} \cdot \omega^{2 n}}{n(2 n+1) n !}=\frac{\omega^{2}}{3}-\frac{\omega^{4}}{20}+\cdots
$$

intendendo qui con $f(\omega)$ una funzione che differisce, dalla prima introdotta, per una costante e per il segno, scelta in modo che:

$$
f(0)=0 ; \quad f(\omega)>0, \quad \text { per } \omega>0 .
$$


I tre termini della $7[1]$ hamno strutture differenti: il primo rappresenta il potenziale dovuto ad una c.c., e si cancella nelle espressioni del nostro "potenziale lacunare" che consiste, come vedremo, di differenze d'espressioni tipo 7[1]. Il secondo termine e indipendente dalla distanza elettrodo-osservatore, e siccome in qualsiasi tipo di misura potenziale vengano misurate d.d.p. nello stesso istante $t$, tale termine non dara alcun contributo. L'unico che contribuira sara quindi il terzo termine che rappresenta il contributo del "potenziale lacunare", riferito all'elettrodo-origine. Se $Q$ e il rapporto tra il potenziale e l'intensita di corrente, si avra per il "contributo lacunare" d'una "corrente a gradino ":

$$
Q=-\frac{x}{\sqrt{\sigma t}} f(\omega)
$$

con $f(\omega)$ data dalla $7[2]$ o dalla $7[3]$, e $(1)$ dalla:

$$
\omega=\Pi r \sqrt{\sigma / t} \text {. }
$$

Nel sistema elettrostatico per $x$ e $K$ si ha:

$$
x=\frac{10^{-10}}{\mathrm{0} \pi}, \quad K=\frac{10^{-10} \sqrt{\pi}}{5},
$$

oppure:

$$
x=50 \frac{\sqrt{10}}{\pi}, \quad K=10^{-3} \mid \frac{\pi}{10} .
$$

Nella $7[5], r$ e misurata in im., $\sigma$ in ohm.m.-1, $Q$ in micro-ohm (cioe $\psi$ in micro-olm e I in Ampère).

In pratica, di norma (1) è talmente piccolo che si può sostituire $f(\omega)$ nella $7[3]$ col suo primo termine di sviluppo $\omega^{2} / 3$, nel qual caso $Q$ diventa:

$$
Q=-x \frac{K^{2}}{3} \cdot \frac{r^{2}}{t \sqrt{t}} .
$$

In realta si misurano spesso potenziali medi tra due istanti $t_{1}$ e $t_{2}$ e cioè:

$$
\bar{Q}_{1,2}=\frac{1}{i_{2}-i_{1}} \cdot \int_{i_{1}}^{t_{2}} Q d t
$$

Passiamo qui alla valutazione dell'integrale:

$$
\int_{i_{1}}^{t_{2}} Q d t
$$


Se $\omega_{1}$ e $\omega_{2}$ rappresentano le $\omega$ corrispondenti a $t_{1}$ e $t_{2}$, tenendo presente che in virtù della $7[5]$

$$
t=K^{2} r^{2} \sigma / \omega^{2}, \quad d t=\left[-2 K^{2} r^{2} \sigma / \omega^{3}\right] d \omega,
$$

si avra:

$$
\begin{gathered}
\int_{i_{1}}^{2} Q d t--\frac{2 x}{\sqrt{\sigma}}\left[\sqrt{t_{1}} F\left(\omega_{1}\right)-\sqrt{t_{2}} F\left(\omega_{2}\right)\right] \\
F(\omega)=\omega \int_{0}^{\omega} f(\omega) \frac{d \omega}{\omega^{2}} .
\end{gathered}
$$

Per ricavare la $F(\omega)$ ricordiamo intanto le seguenti formule che si ottengono facilmente con integrazioni per parti:

$$
\begin{aligned}
& \left\{\frac{d \omega}{\omega^{2}}=-1 / \omega, \quad \int \frac{d \omega}{\omega^{2}} \lg \omega=-\frac{1}{\omega} \lg \omega-\frac{1}{\omega},\right. \\
& \int \frac{d \omega}{\omega^{2}} \int_{\omega^{2}}^{\infty} \frac{e^{-x}}{x} d x=-\frac{1}{\omega} \int_{\omega^{2}}^{\infty} \frac{e^{-x}}{x} d x-2 \int \frac{d \omega}{\omega^{2}} e^{-\omega^{2}} d \omega, \\
& 2 \int \frac{d \omega}{\omega^{3}} \int_{0}^{\omega} e^{-x} d x=-\frac{1}{\omega)^{2}} \int_{0}^{\omega} e^{-x^{2}} d x+\int \frac{d \omega}{\omega^{2}} e^{-\omega^{2}} d \omega, \\
& \int \frac{d \omega}{\omega^{2}} e^{-\omega^{2}} d \omega=-\frac{1}{\omega} e^{-\omega^{2}}-2 \int e^{-\omega^{2}} d \omega .
\end{aligned}
$$

Da queste si ricava immediatamente per $F(\omega)$ :

$$
F(\omega)=-2 \lg (\omega)+e^{-\omega^{2}}-\int_{\omega^{2}}^{\infty} \frac{e^{-x}}{x} d x-\left(\frac{1}{\omega}-2 \omega\right) \int_{0}^{\infty} e^{-x^{2}} d x+\gamma \cdot 7[10]
$$

Dai noti sviluppi delle funzioni che compaiono nella $7[10]$, e ancora piu semplicemente dalle $7[9]$ e 7 [3], si ricava anche lo sviluppo in serie di Taylor della $F(\omega)$ :

$$
F(\omega)=\sum_{1}^{\infty} \frac{(-1)^{n-1} \cdot \omega^{2 n}}{n\left(4 n^{2}-1\right) n !}=\frac{\omega^{2}}{3}-\frac{\omega^{1}}{60}+\ldots
$$

Si ha quindi:

$$
\vec{Q}_{1,2}=-\frac{2 x}{\sqrt{\sigma}} \cdot \frac{1}{t_{2}-t_{2}}\left[\sqrt{t_{1}} F\left(\omega_{1}\right)-\sqrt{t_{2}} F\left(\omega_{2}\right)\right],
$$

con $F(\omega)$ data dalle $7[10]$ e $7[11]$. 
Se come arviene in pratica $\omega$ e piccolo in maniera da sostituire $F(\omega)$ con $\omega-/ 3$, la $7[12]$ diventa:

$$
\bar{Q}_{1,2}=-\frac{2}{3} x K=\sqrt{\sigma} r^{2} / \sqrt{t_{1} t_{2}}\left(\sqrt{t_{1}}+\sqrt{t_{2}}\right)
$$

Si noti che la caratteristica del "potenziale lacunare", in queste condizioni, e l'aumentare in valore assoluto proporzionalmente con il quadrato della distanza (le formule, ripetiamo, si riferjscono a d.d.p. misurate tra un punto a distanza $r$ e l'elettrodo stesso).

Passiamo a considerare un impulso di durata $\tau$, in cui il potenziale viene misurato ad un tempo $t$, dopo la cessazione dell'impulso stesso. Per dedurre in questo caso il "potenziale lacunare" basta applicare le nostre formule per $t+\tau$ e $t$, e sottrarle (potendosi considerare un impulso di durata $\tau$ quale sovrapposizione di due correnti a gradino della stessa intensita, di cui una positiva e una negativa, sfasate tra loro di $\tau$ ). Se $Q$ e il rapporto del "potenziale lacunare» (misurato rispetto all'elettrodo) e l'intensita di corrente, si ha dalla 7[4]:

$$
Q=\frac{x}{\sqrt{\sigma}}\{f(\omega) / \sqrt{t}-f(\omega) / \sqrt{t+\tau}\}
$$

dove

$$
\omega=K r \sqrt{\sigma / t}, \quad \bar{\omega}=K r \sqrt{\sigma /(t+\tau)}=\omega \sqrt{t /(t+\tau)} \cdot \quad 7[15]
$$

Se invece si misura il potenziale medio nei tempi tra $t_{1}$ e $t_{\text {o }}$ successivi all'impulso, si ottiene dalla $7[12]$ per l'analogo rapporto $\bar{Q}_{12}$ :

$$
\begin{gathered}
\bar{Q}_{1,2}=\frac{2 x}{\sqrt{\sigma}} \cdot \frac{]}{t_{2}-t_{1}}\left[\sqrt{t_{1}} F\left(\omega_{1}\right)-\sqrt{t_{2}} F\left(\omega_{2}\right)-\sqrt{t_{1}+\tau F}\left(\bar{\omega}_{1}\right)+\right. \\
\left.+\sqrt{t_{2}+\tau} F\left(\bar{\omega}_{2}\right)\right]
\end{gathered}
$$

dove:

$$
\begin{array}{ll}
\omega_{1}=\pi r \sqrt{\sigma / t_{1}} \quad ; \quad \omega_{2}=\pi r \sqrt{\sigma / t_{2}} \\
\bar{\omega}_{1}=\pi r \sqrt{\sigma / t_{1}+\tau} ; \quad \bar{\omega}_{2}=\pi r \sqrt{\sigma /\left(t_{2}+\tau\right)} .
\end{array}
$$

Molto spesso il potenziale si misura per un tempo uguale a quello dell'impulso, per cui posto $t_{1}=t, t_{2}=t+\tau$, la $7[16]$ può seriversi:

$$
\bar{Q}=\frac{2 x}{\tau \sqrt{ } \sigma}\left\{\sqrt{t} F(\omega)-2 \sqrt{t+\tau} F^{\prime}(\bar{\omega})+\sqrt{t+2 \tau} F(\bar{\omega})\right\}
$$


con:

$$
\begin{gathered}
\omega=K r \sqrt{\sigma / t}, \quad \bar{\omega}=K r \sqrt{\sigma /(t+\tau)}=\omega \sqrt{t /(t+\tau)} \\
\bar{\omega}=K r \sqrt{\frac{\sigma}{1+2 \tau}}=\omega \sqrt{\frac{t}{t+2 \tau}} .
\end{gathered}
$$

Ise $7[14], 7[16], 7[18]$, praticamente quando si possa sostituire $f(\omega)$ e $F(\omega)$ con $\omega^{2} / 3$, diventano:

$$
\begin{array}{cc}
Q=\frac{x}{3} \pi^{\cdot} r^{2}\left(t^{-3 / 2}-\left(t+\tau^{3}\right)^{-1 / 2}\right], & 7[20] \\
\bar{Q}_{1,2}=\frac{2}{3} w \pi^{-} v \sqrt{u} \frac{r^{2}}{t_{2}-t_{1}}\left[t_{1}^{-1 / 2}-t_{2}^{-1 / 2}-\left(\tau+t_{1}\right)^{-1 / 2}+\left(\tau+t_{2}\right)^{-1 / 2}\right], & 7[21] \\
Q=\frac{2}{3} x K^{2} \sqrt{\sigma r^{2}} \tau^{-1}\left[t^{-1 / 2}-2(t+\tau)^{-1 / 2}(1+2 \tau)^{-1 / 2}\right] . & 7[22]
\end{array}
$$

Eseguendo ad es. misure Wenner, basta sostituire nelle $7[12], 7[16]$ e $7[18]$ la

e la

$$
f(\omega) \quad \operatorname{con} 2[f(\omega)-f(2 \omega)]
$$

$$
F(\omega) \quad \operatorname{con} 2[F(\omega)-F(2 \omega)]
$$

Questo significa che le $7[20], 7[21], 7[22]$ vanno moltiplicate per -6 infatti:

$$
2\left[\omega^{2} / 3- \pm \omega^{2} / 3\right]=-2 \omega^{2}=-6 \omega^{2} / 3
$$

Ne deriva che col metodo Wenner il "potenziale lacunare" nella nostra teoria risulta negativo.

Successione periodica d'impulsi.

Passiamo finalmente a considerare una successione periodica di impulsi + e - .

Sia $T$ il tempo decorrente tra un impulso positivo e uno negativo (semiperiodo), ogni impulso abbia una durata $\tau=(1-\alpha) T / 2$, la misura avvenga dopo un tempo aT/2 dalla fine dell'ultimo impulso positivo, e la misura del potenziale avvenga anche essa per un tempo $(1-a) T / 2(\alpha$ è cioè $<1$ : in pratica di solito $\alpha=1 / 5)$.

Per ottenere $\bar{Q}$ in queste condizioni occorre sommare i contributi dei singoli impulsi dati dalla $7[19]$. Secondo questa formula l'ennesimo 
impulso (contando l'ultimo come 0 ) con segno $(-1)^{n}$ e sostituendo $\tau$ con $(1-a) T / 2, t$ con $a T / 2+n T$, si ottiene per questo contributo:

$$
\begin{aligned}
\bar{Q}_{n}=4 x(-1)^{n}\left\{(2 n+a)^{1 / 2} F\left(\frac{v}{\sqrt{2 n+a}}\right)-2(2 n+1)^{1 / 2} F\left(-\frac{v}{\sqrt{2 n+1}}\right)+\right. \\
+[2(n+1)-a]^{1 / 2} F\left(\frac{v}{\sqrt{2 n+1}}\right) ! /(1-a) \sqrt{2 \sigma T}, \quad 7[23]
\end{aligned}
$$

ove si e posto:

$$
v=\pi r \sqrt{2 \sigma / T}
$$

Sommando i contributi dei diversi impulsi si arriva a:

$$
\begin{aligned}
\bar{Q} & \left.=\frac{2 u}{1-a}\right] \frac{2}{\sigma T}\left(v u T\left(\frac{v}{\sqrt{a}}\right)-2 \sum_{n=0}^{\infty}(-1)^{n} \sqrt{2 n+1} F\left(\frac{v}{\sqrt{2 n+1}}\right)+\right. \\
& +\sum_{n=1}^{\infty}(-1)^{n}\left[\sqrt{2 n-a} F\left(\frac{v}{\sqrt{2 n-a}}\right)-\sqrt{2 n+a F}\left(\frac{v}{\sqrt{2 n+a}}\right)\right\} \cdot 7[25]
\end{aligned}
$$

Nella realtà si è detto che si può sostituire al posto di $F(\omega)$ il termine $\omega^{2} / 3$, e con cio la $7[25]$ diventa:

$$
\left.\bar{Q}=\frac{4 x K^{2} \sqrt{2 \sigma}}{3(1-\alpha)} !^{-1 / 2}-2 \mathrm{~A}+\sum_{n=1}^{\infty}(-1)^{n}\left[\left(2^{n}-\alpha\right)^{-1 / 2}-(2 n+a)^{-1 / 2}\right]\right) \frac{\tau^{2}}{\sqrt{x^{1 / 3}}}
$$

dove:

$$
1=\sum_{0}^{\infty} \frac{(-1)^{n}}{\sqrt{2 n+1}}=-1-\frac{1}{\sqrt{3}}+\frac{1}{\sqrt{5}}-\cdots
$$

Inoltre nelle misure pratiche $a$ e sufficientemente piccolo da poter porre:

$$
(2 n-a)^{-1 / 2}-(2 n+a)^{-1 / 2} \cong a / 2 \sqrt{2} \sqrt{n^{3}},
$$

il che significa trascurare nella parentesi grandezze dell'ordine $(\alpha / 2)^{3}$. Infatti, di solito, nelle misure si ha $a=1 / 5$, il che comporta un errore dell'ordine di grandezza di un millesimo. In quest'ipotesi la 7 [26] diventa:

$$
\bar{Q}=\frac{16 x K^{2}}{3(1-\alpha)} \sqrt{\alpha}\left(a^{-1 / 2}-2 A+\alpha B / 2 \sqrt{2}\right) r^{2} f^{3 / 2},
$$

ove:

$$
B=\sum_{1}^{\infty} \frac{(-1)^{n-1}}{n \sqrt{n}}=1-\frac{1}{2 \sqrt{2}} \cdot \frac{1}{3 \sqrt{3}}-\ldots
$$

daundo alla frequenza il valore:

$$
f=1 / 2 T .
$$


Se le misurazioni si effettuano con la modalita Wenner, la 7[29] va ancora moltiplicata per -6 , come e stato fatto presente per l'impulso singolo, e si avra:

$$
\bar{Q}_{\omega}=-\frac{c \sqrt{\sigma}}{1-\alpha}\left[\alpha^{-1 / 2}-2 A+\alpha B / 2 \sqrt{2}\right] r^{2} f \sqrt{f},
$$

con $c=32 x K^{\circ}$.

Il "potenziale lacunare" assume, in queste condizioni, valori negativi che crescono, in valore assoluto, proporzionalmente al quadrato della distanza e alla potenza $3 / 2$ iella frequenza.

Se confrontiamo questi risultati con quelli riportati dall'Evjen, si nota anzitutto che nella sua tabella compaiono sia dati positivi che dati negativi, il che dimostrerebbe, secondo questo A., l'esistenza di una riflessione, cioe i dati non si riferirebbero ad un terreno omogeneo. Per ora non possediamo un'esatta teoria della riflessione, ma si puo supporre che il suo effetto diminuira con la distanza, e decrescera o crescera piuttosto lentamente con la frequenza. In altre parole con l'aumentare della distanza e della frequenza, i risultati si avvicineranno a quelli dati dalla 7[30] (il che si nota effettivamente nella tabella dell'Evjen).

Mentre per "basse frequenze e distanze " la $\vec{Q}_{\omega}$ misurata risulta positiva - il che è indice che predomina l'effetto di riflessione su uno strato ad una certa profondita, col crescere dell'una o dell'altra grandezza la $Q$ diventa negativa, segno che predomina per così dire leffetto primario, cioe quello dovuto solamente ad un terreno omogeneo.

Inoltre per piu alti valori di frequenza e distanza, riportati nella sucitata tabella, l'andamento in funzione di $r$ ed $f$, e quello richiesto dalla formula $7[30]$.

Faremo ancora un confronto qualitativo: le costanti $A$ e $B$, date dalle $7[26]$ e $7[30]$, si trovano sommando le serie con un procedimento - di cui non citiamo i particolari - dando i seguenti risultati:

$$
A=0,668 ; \quad B=0,665 \text {. }
$$

Per la costante $C$, per le $7\left[5^{\prime \prime}\right]$ si ha pure:

$$
C=5,06 \cdot 10^{-1} \text {. }
$$

Posto inoltre:

$$
\alpha=1 / 5
$$

la $7[30]$ diventa approssimativamente:

$$
\vec{Q}_{\omega}=c\left(\frac{r}{100}\right)^{2} f \sqrt{f s} \text { microhom. }
$$


Il confronto di questa $\bar{Q}_{\omega}$ coi dati dell'Evjen comporterebbe un valore piuttosto grande per la conduttivita $\left(\sim 10^{-1}\right)$, il che fa supporre (a meno che il valore $10^{-5}$ per la $\sigma$ citato dall'Evjen non sia scorretto) che all'effetto riflessivo di strato se ne sovrapponga un altro dello stesso tipo di quello dato dalla $7[30]$. Siccome qui si tratta essenzialmente di un "effetto di cariche che insorgono alla superficie terrestre", e probabile che un simile effetto (cioe dello stesso segno e che segue un'analoga legge per distanza e frequenza), sia provocato dalla superficie che separa lo strato riffettente.

Da cio deduciamo che all'inserimento d'uno strato consegue una legge piuttosto complicata, e che probabilmente per piccole distanze e frequenze, ei si trova di fronte ad un evento simile ad una riflessione: mentre col crescere della distanza e frequenza ne predomini un altro causato dalla formazione di cariche sulla superficie di separazione, del tutto simile all'effetto della superficie terrestre.

\section{BIBLIOG RAFIA}

(1) A. Belluigi, Sul metodo geoelettrico El/lex per la ricerca diretla del petrolio. "Boll. Servizio Geol. d'Italia ", Roma, 1955.

(2) A. Belluigi, Sull'effetto e.m. diretto di emittori alternativi in un terreno omogeneo. "Annali di Geofisica", Roma, 1954.

( $\left.{ }^{3}\right)$ A. Belluigi, La ricerca dell'acqua tellurica. Roma, "Convegno sull'approvvigionamento idrico della Capitalen. Maggio, 1957.

$\left({ }^{4}\right)$ A. Belluigi, Teoria approssimata della P.P. "Annali di Geofisica ", Roma, 1956.

(5) C. W. Horton, On the use of e.m. Waves in Geoph. Prospecting. "Geophysics ", 1946.

$\left(^{6}\right)$ W. Bradlex Lewis, Electric Field of a oscillating Dipole. "Geophysies ", 1946.

(7) H. M. EvJen, Theory and Practice of Low - Frequency e.m. Exploration. "Geophysies ", 1948.

$\left.{ }^{8}{ }^{8}\right)$ H. M. EvJen, Surface Electrical Method for Detect Oil Directly. "World Oil ", February 1953. 